\title{
Kinematic turbulent dynamo in the large Prandtl number regime
}

\author{
R. J. West ${ }^{1}$, S. Nazarenko ${ }^{1}$, J.-P. Laval ${ }^{2}$, and S. Galtier ${ }^{3}$ \\ ${ }^{1}$ Mathematics Institute, University of Warwick, Coventry CV4 7AL, UK \\ 2 Laboratoire de Mécanique de Lille, CNRS, UMR 8107, Bld. Paul Langevin, 59655 Villeneuve d'Ascq Cedex, France \\ ${ }^{3}$ Institut d'Astrophysique Spatiale, Université de Paris-Sud, CNRS, UMR 8617, Bât. 121, 91405 Orsay Cedex, France
}

Received 21 May 2003 / Accepted 21 October 2003

\begin{abstract}
We present the results of a numerical investigation of the turbulent kinematic dynamo problem in a high Prandtl number regime. The scales of the magnetic turbulence we consider are far smaller than the Kolmogorov dissipative scale, so that the magnetic wavepackets evolve in a nearly smooth velocity field. Firstly, we use a stochastic Euler-Maruyama method to simulate the Kraichnan-Kazantsev model (KKM) in which the strain matrix is taken to be independent of coordinate and Gaussian white in time. We test the theoretical predictions for the growth of rates of the magnetic energy and higher order moments (Kazantsev 1968; Chertkov et al. 1999; Kulsrud \& Anderson 1992), the shape of the energy spectrum (Schekochihin et al. 2002a; Kazantsev 1968; Nazarenko et al. 2003; Kulsrud \& Anderson 1992) and the behavior of the polarization and spectral flatness, new measures introduced in Nazarenko et al. (2003). In general, the results appear to be in good agreement with the theory, with the exception that the predicted decay of the polarization in time is not reproduced well in the stochastic numerics. Secondly, in order to study the sensitivity of the KKM predictions to the choice of strain statistics, we perform additional simulations for the case of a Gaussian strain with a finite correlation time and also for a strain taken from a DNS data set. These experiments are based on non-stochastic schemes, using a timestep that is much smaller than the correlation time of the strain. We find that the KKM is generally insensitive to the choice of strain statistics and most KKM results, including the decay of the polarization, are reproduced well. The only exception appears to be the flatness whose spectrum is not reproduced in accordance with the KKM predictions in these simulations.
\end{abstract}

Key words. galaxies: magnetic fields - ISM: magnetic fields - magnetic fields - methods: numerical - MHD - turbulence

\section{Introduction}

Many astrophysical magnetic fields are believed to be generated via a dynamo action (Moffatt 1978; Parker 1979; Childress \& Gilbert 1995) in systems where the magnetic field diffusivity $K$ is much less than that of the kinematic viscosity $v$. These systems correspond to flows with large magnetic Prandtl number $\operatorname{Pr}=v / \kappa$. Such astrophysical situations are observed in the interstellar medium as well as in protogalactic plasma clouds where the magnetic Prandtl number varies respectively from $P r \sim 10^{14}$ to $P r \sim 10^{22}$ (Chandran 1998; Kulsrud 1999; Schekochihin et al. 2002a). Such high values of $P r$ lead to an interesting interval of scales (from 7 to 11 decades in our examples) below the viscous cut-off, but above the magnetic diffusive scale, where magnetic fluctuations are stretched by a randomly changing smooth velocity field. The small-scale kinematic dynamo problem can thus be formulated as to whether a small initially "seeded" magnetic field, subject to stretching by a prescribed smooth velocity field, will grow in time.

One can picture the evolution of an ensemble of magnetic wavepackets, each traveling together with the fluid particles

Send offprint requests to: S. Nazarenko,

e-mail: snazar@maths.warwick.ac.uk which are distorted by the local strain. By assuming a given form of the strain statistics, an idealized homogeneous and isotropic strain that is a Gaussian white noise process, one can simplify the problem, leading to a productive framework from which analytical results can be obtained. This formalism is known as the Kraichnan-Kazantsev model (KKM) (Kraichnan \& Nagarajan 1967; Kazantsev 1968). It should be remembered that such a model is a simplification and a real turbulent velocity field will exhibit finite correlations of the fluid velocity field, as well as being to some degree non-Gaussian, inhomogeneous and anisotropic. Therefore, in this numerical investigation we will also consider more realistic examples of the velocity field.

The work presented here is based on a kinematic model, making use of the linear induction equation of the magnetic field $\boldsymbol{B}(\boldsymbol{x}, t)$

$D_{t} \boldsymbol{B}=\boldsymbol{B} \cdot \nabla \boldsymbol{v}+\kappa \nabla^{2} \boldsymbol{B}$

where $D_{t} \equiv \partial_{t}+\boldsymbol{v} \cdot \nabla$ is the material derivative, $\boldsymbol{v}$ the velocity, and $\kappa$ the magnetic diffusivity. Physically, we can have in mind the following picture for the evolution of our dynamo system. Initially, for small $\kappa$, the $\boldsymbol{B}$-field will be "frozen" into the flow behaving as a passive vector field (Soward 1994). After some period of time, and hence growth of the magnetic field, diffusion will become important and will reduce this rate of growth. 
In particular, using the KKM the growth of the total mean magnetic energy $<\boldsymbol{B}^{2}(t)>$ was obtained by Kazantsev (1968) and that of the higher order moments $\left\langle\boldsymbol{B}^{2 n}(t)>\right.$ by Chertkov et al. (1999). Schekochihin et al. (2002a) have extended this work to include the effects of compressibility.

The work presented here has two primary aims. The first aim is to numerically verify the analytical scaling laws found by Kazantsev (1968) and Chertkov et al. (1999) for the even moments $\left\langle\boldsymbol{B}^{2 n}(t)>\right.$ (where $n$ is a positive integer) in both the perfect conductor (non-diffusive) and diffusive regimes. We will also compute the Fourier space one-point correlators which have recently been investigated analytically (Schekochihin et al. 2002a; Nazarenko et al. 2003). In the past much emphasis has been placed solely on investigations of the magnetic energy spectrum. However, higher order correlators are also of significance. Indeed, it has been shown that the new quantities, the mean polarization of the magnetic turbulence and the spectral flatness, are of particular interest as they characterize the properties of the small-scale intermittency (Nazarenko et al. 2003). The polarization (not to be confused with the helicity!) measures how "squashed" the magnetic wavepackets are, whereas the flatness measures the degree of "noisiness" of the magnetic energy spectrum (see Sect. 3 for the mathematical definitions of these quantities). These newer theoretical objects will also be investigated numerically. Our second aim is to study the sensitivity of the dynamo model to the choice of strain statistics. In a quest to test the universality of the analytical results found using the KKM, we will consider the case of a finite-correlated Gaussian strain field. We will also consider a more realistic strain field generated from a DirectNumerical-Simulation (DNS) of the Navier-Stokes equations.

The presentation of this paper has been organized into six main sections. In the next section we give a brief introduction to the small-scale dynamo problem, with a summary of the KKM analytical results as derived by Chertkov et al. (1999) and Kazantsev (1968). In the third section we outline some of the new KKM analytical results in regard to the behavior of the one-point Fourier space correlators; a more detailed discussion of which can be found in Nazarenko et al. (2003). The fourth section provides an overview of the numerical method used to investigate the KKM and its subsequent results. In the fifth section we outline the method and results of the finite-correlated Gaussian and DNS based investigations. Finally, we draw our conclusions in the sixth section.

\section{Moments of $B$}

Let us briefly summarize the analytical results for the moments of $\boldsymbol{B}(t)$ obtained within the KKM by Chertkov et al. (1999). At scales below the viscous cut-off Batchelor (Batchelor 1950, 1954) argued the velocity field would be random and smooth (Batchelor regime). In MHD a uniform velocity cannot alter a system's magnetic field. The flow

$v_{i}=\sigma_{i j} x_{j}$

where $\sigma(t)$ is a co-ordinate independent random strain matrix, represents one of the simplest choices of a velocity field that allows for the transfer of kinetic energy into magnetic energy (Zel'dovich et al. 1984). The incompressibility condition $\nabla \cdot v=0$ in this case corresponds to $\operatorname{Tr}[\sigma]=0$ for all time. It is natural to reformulate our mathematical description in terms of Lagrangian dynamics (namely following particles) in which case $D_{t} \equiv \mathrm{d} / \mathrm{d} t$ in the induction Eq. (1). Zel'dovich et al. (1984) (Soward 1994) showed that given an initial condition, the induction Eq. (1) can be re-written in Fourier space as

$\boldsymbol{B}(\boldsymbol{k}, t)=J(t) \boldsymbol{B}(\boldsymbol{q}, 0) \exp \left(-\kappa \int_{0}^{t} k^{2}\left(t^{\prime}\right) \mathrm{d} t^{\prime}\right)$,

where the Jacobian $J(t)$ satisfies

$\frac{\mathrm{d}}{\mathrm{d} t} J=\sigma J$

with the initial condition $J(0)=I$, with $I$ the unit matrix, and $\boldsymbol{q}=\boldsymbol{k}(0)$ is the initial wavevector related to $\boldsymbol{k}(t)$ by

$\boldsymbol{q}=J^{T} \boldsymbol{k}$.

Moments of $\boldsymbol{B}$ are calculated via two independent averagings, one over initial statistics, and the other over various realizations of the strain matrix (Chertkov et al. 1999). The initial $\boldsymbol{B}$ field is assumed to be homogeneous, isotropic and Gaussian, with zero mean and the following variance

$<B_{a}(\boldsymbol{k}, 0) B_{b}\left(\boldsymbol{k}^{\prime}, 0\right)>=\left(\delta_{a b}-\frac{k_{a} k_{b}}{k^{2}}\right) k^{2} E_{m} \mathrm{e}^{-k^{2} L^{2}} \delta\left(\boldsymbol{k}-\boldsymbol{k}^{\prime}\right)$,

where $L$ is the length-scale at which the "seed" magnetic noise is initially concentrated and $E_{m}$ is a constant which determines the initial turbulence intensity. After averaging over the initial statistics Chertkov et al. (1999) obtained the following expression for $\boldsymbol{B}^{2}(t)$,

$\boldsymbol{B}^{2}(t)=\int E_{m} \mathrm{e}^{-q^{2} L^{2}} \mathrm{e}^{-2 \kappa \boldsymbol{q} \Lambda \boldsymbol{q}} q^{2} J_{i a}\left(\delta_{a b}-\frac{q_{a} q_{b}}{q^{2}}\right) J_{i b} \mathrm{~d} \boldsymbol{q}$,

with summation over repeated indices and where $q=|\boldsymbol{q}|$ and $\Lambda(t)$ satisfies

$\Lambda(t)=\int_{0}^{t} \mathrm{~d} t^{\prime} J^{-1}\left(t^{\prime}\right) J^{-T}\left(t^{\prime}\right)$.

The full even moments $<\boldsymbol{B}^{2 n}(t)>$ where calculated by taking the value of $\boldsymbol{B}^{2}$ to the correct power and then averaging over the realizations of the velocity field. For an isotropic, deltacorrelated, Gaussian strain field

$<\sigma_{a b}(t) \sigma_{a b}(0)>=10 \lambda_{1} \delta(t)$

where $\lambda_{1}$ is the growth rate of a material line element, corresponding to the largest Lyapunov exponent of the flow, Chertkov et al. (1999) derived the following expressions for the scaling behavior of the even moments $<\boldsymbol{B}^{2 n}(t)>$. In the perfect conductor regime $t<3 t_{\mathrm{d}} /(2 n+3)$, we have

$<\boldsymbol{B}^{2 n}(t)>\simeq \mathrm{e}^{2 \lambda_{1} n(2 n+3) t / 3}$,

where on dimensional grounds the scale at which magnetic diffusion becomes important is $r_{\mathrm{d}} \simeq \sqrt{\kappa / \lambda_{1}}$ and the corresponding time is $t_{\mathrm{d}} \simeq \lambda_{1}^{-1} \ln \left(L / r_{\mathrm{d}}\right)$. In the diffusive regime $t>3 t_{\mathrm{d}} /(n+2)$, we have

$<\boldsymbol{B}^{2 n}(t)>\simeq \mathrm{e}^{\lambda_{1} n(n+4) t / 4}$.

For $n=1$, this result was derived earlier by Kazantsev (1968). 


\section{Fourier space correlators}

Traditionally, Fourier space analysis of the dynamo problem has only really involved investigations of the $k$-space correlator corresponding to the energy spectrum $E(k, t)=\left\langle|\boldsymbol{B}(\boldsymbol{k})|^{2}\right\rangle$ (Kazantsev 1968; Kulsrud \& Anderson 1992; Schekochihin et al. 2002a). Kazantsev (1968) studied the dynamo system as an eigenvalue problem from which he was able to obtain the growth exponents of the total magnetic energy. The evolution of the energy spectrum in $k$-space has also been studied both analytically and numerically by Kulsrud \& Anderson (1992).

In this KKM analysis, the way in which we define the incompressible strain matrix is different to (9) used in Chertkov et al. (1999). We write

$\frac{\sigma_{i j}}{\sqrt{\Omega}}=G_{i j}-\frac{\operatorname{Tr}[G]}{3} \delta_{i j}$

where $G$ is a $3 \times 3$ matrix made up of independent Gaussian $N(0 ; 1)$ random variables, normally distributed (i.e. Gaussian) with zero mean and a standard deviation of 1 , such that

$<G_{i j}(t) G_{k l}(0)>=\delta_{i k} \delta_{j l} \delta(t)$.

This choice is motivated by its convenience in numerical modeling. One should note there is a degree of flexibility in how we define our strain statistics, a more detailed discussion of which can be found in the Appendix. The KKM results remain the same for different choices of the strain statistics, the only difference being that time is re-scaled by a constant factor.

\subsection{Energy spectrum}

\subsubsection{Energy spectrum: $\kappa=0$ solution}

In the limit of zero diffusion $\kappa=0$, the 3D energy spectrum has the solution (Kulsrud \& Anderson 1992; Schekochihin et al. 2002a; Nazarenko et al. 2003)

$E(k, t)=\frac{E_{0}}{\sqrt{t}}\left(\frac{k}{q}\right)^{-1 / 2} \exp \left(-\frac{3 \ln ^{2}(k / q)}{4 \Omega t}\right) \exp \left(\frac{5 \Omega t}{4}\right)$,

where $q$ is the wavenumber at which $E(k)$ is initially concentrated at $t=0$, and $E_{0}$ is a constant depending on the initial conditions. Here, $\Omega$ is a constant equal to the rms of the strain, see definition (12). If we let $t_{\mathrm{d}}$ be the diffusion time as defined in Sect. 2 or Chertkov et al. (1999), then for large time $\Omega^{-1} \ln ^{2}(k / q) \ll t \ll t_{\mathrm{d}}$, i.e. in the perfect conductor regime, we have ${ }^{1} E \sim k^{-1 / 2}$. Alternatively, we can think of the $k^{-1 / 2}$ slope as being present over an interval of wavenumber space $k_{c-} \ll k \ll k_{c+}$ where

$k_{c \pm}(t) \sim \exp ( \pm \sqrt{4 \Omega t / 3})$.

The critical wavenumbers $k_{c+}$ and $k_{c-}$ govern when the exponential log squared term above becomes important. We see the fronts at $k_{c+}$ and $k_{c-}$ propagate exponentially in time, and within this $k^{-1 / 2}$ range the spectrum also grows exponentially.

${ }^{1}$ Here, we are considering a 3D spectrum. Kulsrud \& Anderson (1992) considered the equivalent 1D spectrum which has a corresponding $k^{3 / 2}$ scaling regime.
By integrating Eq. (14), over the whole of wavenumber space, one can obtain the growth rate of the total magnetic energy

$$
<B^{2}(t)>=\int E(k, t) \mathrm{d} \boldsymbol{k} \simeq \text { const } \exp \left(\frac{10 \Omega t}{3}\right)
$$

which agrees with result (10) for $n=1$ when we take into account the slightly different definitions of the strain co-variance. Indeed, one finds that the $\lambda_{1} t$ of (10) and $\Omega t$ of (16) are equivalent if we re-scale time by a factor of $4 / 5$. Hence the scaling laws of Chertkov et al. (1999), presented in Sect. 2, for the diffusive regime should be re-written for our choice of strain statistics as

$<B^{2 n}(t)>\simeq \mathrm{e}^{2 n \Omega(2 n+3) t / 3}$.

Further, in the diffusive regime we have

$<B^{2 n}(t)>\simeq \mathrm{e}^{\Omega n(n+4) t / 4}$.

\subsubsection{Energy spectrum: $\kappa \neq 0$ solution}

If all wavevectors are initially of the same length $|q|$, and we are interested in large time asymptotics (Kazantsev 1968; Kulsrud \& Anderson 1992; Schekochihin et al. 2002a; Nazarenko et al. 2003), the solution in the diffusive regime takes the form

$E(k, t) \simeq$ const $\mathrm{e}^{5 \Omega t / 4} k^{-1 / 2} t^{-3 / 2} K_{0}\left(k / k_{\kappa}\right)$,

where $K_{0}$ is a MacDonald function of zeroth order and $k_{\kappa}$ is a constant given by $k_{\kappa}=\sqrt{\Omega / 6 \kappa}$. In fact this $k_{\kappa}$ is the wavenumber at which diffusion becomes important. It is therefore equivalent to the estimated $k_{\mathrm{d}} \sim 1 / r_{\mathrm{d}}$ derived on purely dimensional grounds in Sect. 2.

It is now interesting to discuss the critical value of the initial Reynolds number for the dynamo process, predicted by Novikov et al. (1983) to be about 60 and recently confirmed via DNS in Branderburg et al. (1995). It turns out that there is no critical value in the Kazantsev-Kraichnan model. This follows from formula (19) which is the $t \rightarrow \infty$ limit for any initial condition (the initial condition affects only the constant prefactor). This is easy to see by taking the large time limit in the general solution given in Nazarenko et al. (2003). This might seem surprising because intuitively one would expect initial fields placed far below the dissipation scale to dissipate. However, the $k$-space diffusion leads to penetration of some energy above the dissipative scale, it growth there, and penetration back under the dissipative scale in an amplified form. Such a "positive feedback loop" qualitatively explains the mathematical result for the asymptotic growth given by (19). Thus, to describe the result on the critical Reynolds number, the Kazantsev model would not be sufficient and would have to be generalized.

\subsection{Flatness and polarization}

Although the energy spectrum solutions described above provides us with some very useful information, they do not provide us with a complete picture of our system. In the recent work of Nazarenko et al. (2003), higher one-point correlators of the 
magnetic field in Fourier space were studied ${ }^{2}$. In this paper, as well as for the energy spectrum $E(k, t)$, we will also study two other correlators

$$
\begin{aligned}
& S(k, t)=<|\boldsymbol{B}(\boldsymbol{k})|^{4}>, \\
& T(k, t)=<\left|\boldsymbol{B}^{2}(\boldsymbol{k})\right|^{2}>.
\end{aligned}
$$

Let us briefly review the results from Nazarenko et al. (2003) regarding these correlators.

\subsubsection{Polarization spectrum and flatness: $\kappa=0$ solutions}

In the perfect conductor regime, the fourth order correlators of Fourier amplitudes $S \equiv<|\boldsymbol{B}(\boldsymbol{k})|^{4}>$ and $T \equiv<\left|\boldsymbol{B}^{2}(\boldsymbol{k})\right|^{2}>$ have exact solutions

$$
\begin{aligned}
S(k, t)= & \frac{1}{\sqrt{t}}\left(\frac{k}{q}\right)^{1 / 2} \exp \left(-\frac{3 \ln ^{2}(k / q)}{4 \Omega t}\right) \\
& \times\left[V_{0} \exp \left(\frac{21 \Omega t}{4}\right)+\frac{3}{7} Q_{0} \exp \left(-\frac{3 \Omega t}{4}\right)\right], \\
T(k, t)= & \frac{1}{\sqrt{t}}\left(\frac{k}{q}\right)^{1 / 2} \exp \left(-\frac{3 \ln ^{2}(k / q)}{4 \Omega t}\right) \\
& \times\left[V_{0} \exp \left(\frac{21 \Omega t}{4}\right)-\frac{4}{7} Q_{0} \exp \left(-\frac{3 \Omega t}{4}\right)\right],
\end{aligned}
$$

where $V_{0}$ and $Q_{0}$ are constants. In large time the $Q_{0}$ terms can be neglected and, as with the energy spectrum, these spectra develop a $k^{1 / 2}$ scaling between two propagating fronts $k_{c-} \ll$ $k \ll k_{c+}$.

Combining these two fourth order correlators, we find an important new quantity $P=(S-T) / S$, the physical significance of which becomes clear when we write $P$ as

$$
\begin{aligned}
P & =\frac{4}{<|\boldsymbol{B}|^{4}>} \sum_{i \neq j}<\left(\mathfrak{I}\left\{B_{i} B_{j}^{*}\right\}\right)^{2}> \\
& =\frac{4}{<|\boldsymbol{B}|^{4}>} \sum_{i \neq j}<\left|B_{i}\right|^{2}\left|B_{j}\right|^{2} \sin ^{2}\left(\phi_{i}-\phi_{j}\right)>,
\end{aligned}
$$

where $\phi_{i}$ is the phase of the complex component $B_{i}$ and the operator $\mathfrak{J}\{\cdot\}$ takes the imaginary part of an expression. In this form we see that $P$ contains information about the phases of the $k$-space modes. $P$ can be thought of as the mean normalized polarization. Indeed, $P=0$ corresponds to the plane polarization of the Fourier modes. In contrast for a Gaussian magnetic field one finds the polarization $P=1 / 3$. Combining (22) and (23), in large time we find the normalized polarization $P$ evolves as

$P=\frac{Q_{0}}{V_{0}} \exp (-6 \Omega t)$

That is, the polarization is independent of wavenumber $k$ and decays exponentially. This solutions tells us that in the perfect conductor regime, the Fourier modes of the magnetic field will

\footnotetext{
${ }^{2}$ These were objects of the form $\left\langle|\boldsymbol{B}|^{2 n}\left|\boldsymbol{B}^{2}\right|^{2 m}>\right.$ which represent a basis for all one-point correlators in magnetic turbulence which is isotropic.
}

eventually become plane polarized. In comparison, we should recall that a Gaussian field has a finite polarization, thus our turbulent magnetic field is far from being Gaussian.

Another important measure of turbulent intermittency in a fluid, both in real and Fourier space, is the flatness. The spectral flatness is defined as the ratio

$F=\frac{S}{E^{2}}=\frac{<|\boldsymbol{B}(\boldsymbol{k})|^{4}>}{<|\boldsymbol{B}(\boldsymbol{k})|^{2}>^{2}}$

Easy to see that $F-1$ measures the "noise" of the energy spectrum. Indeed, the square of the standard deviation of the instantaneous $k$-space energy distribution, $|\boldsymbol{B}(\boldsymbol{k})|^{2}$, is equal to $<|\boldsymbol{B}(\boldsymbol{k})|^{4}>-<|\boldsymbol{B}(\boldsymbol{k})|^{2}>^{2}$ and, therefore, the relative fluctuation squared is $F-1$. Using (26), one finds a Gaussian field has a constant flatness of $F=3 / 2$. On the other hand small-scale intermittency corresponds to a field with a flatness that grows both in time and in wavenumber space. Indeed, in the perfect conductor regime, the magnetic field displays such small-scale intermittent behavior (Nazarenko et al. 2003), with

$F \sim t^{1 / 2} \exp \left(\frac{11 \Omega t}{4}\right) k^{3 / 2} \exp \left(\frac{3 \ln ^{2}(k / q)}{4 \Omega t}\right)$.

For $k_{c-}<k<k_{c+}$, we see there is a range of $k^{3 / 2}$ scaling, while for larger $k$ there is an increase in the flatness arising from the log-squared exponential term. This intermittency in Fourier space can be attributed to the presence of coherent structures, as will be discussed later.

\subsubsection{Polarization spectrum and flatness: $\kappa \neq 0$ solutions}

In the diffusive regime the fourth order correlators $S$ and $T$ have large time solutions (Nazarenko et al. 2003)

$S \simeq k^{1 / 2} t^{-3 / 2}\left[V_{0} \mathrm{e}^{21 \Omega t / 4}+\frac{3}{7} Q_{0} \mathrm{e}^{-3 \Omega t / 4}\right] K_{0}\left(\frac{k}{k_{\kappa, 2}}\right)$,

$T \simeq k^{1 / 2} t^{-3 / 2}\left[V_{0} \mathrm{e}^{21 \Omega t / 4}-\frac{4}{7} Q_{0} \mathrm{e}^{-3 \Omega t / 4}\right] K_{0}\left(\frac{k}{k_{\kappa, 2}}\right)$,

where $k_{\kappa, 2}=\sqrt{\Omega / 12 \kappa}$. This $k_{\kappa, 2}$ can again be interpreted as the wavenumber at which diffusion becomes important. However, in comparison to the energy spectrum we should note that the spectral cut-off will be at a smaller wavenumber as $k_{\kappa, 2}<k_{\kappa}$. Importantly we see that the normalized polarization (25) behaves identically in both the diffusive and perfect conductor regimes. In contrast, the behavior of the flatness is modified

$F=\frac{S}{E^{2}} \simeq k^{3 / 2} t^{3 / 2} \mathrm{e}^{11 \Omega t} \frac{K_{0}\left(k / k_{\kappa, 2}\right)}{\left(K_{0}\left(k / k_{\kappa}\right)\right)^{2}}$,

when compared to (27). For small $k$, we again have a region of $k^{3 / 2}$ scaling but now with a log correction arising from the MacDonald functions. For large $k$, below the spectral cutoff, the additional MacDonald functions act to heighten the 
flatness. That is, the introduction of a finite diffusivity actually increases the small-scale intermittency.

\section{KKM numerical investigation}

The key to modeling the KKM numerically is the successful integration of Eq. (4) to find $J$. As the strain matrix on the right hand side of (4) contains noise, it is evident that this is not a normal ordinary differential equation (ODE) and should instead be interpreted in the stochastic sense. Following the analytical formalism (12) and (13), the elements of the strain matrix $\sigma$ will be made up from a matrix of Gaussian random variables $G$, such that the incompressibility condition $\operatorname{Tr}[\sigma]=0$ is satisfied. As with any stochastic formalism one must decide the form of the integral. Mathematically both the Ito and Stratonovich definitions of the stochastic integral are correct. It is only in a few special cases that both interpretations produce the same solutions. In the present problem this is not the case. Indeed, the Stratonovich formalism is the correct interpretation for our problem as we are using white noise as an idealization of what in reality is a colored noise process (Kloeden et al. 1997).

The simplest numerical scheme one can use to solve Stochastic Differential Equations (SDE) is called the Euler-Maruyama scheme (EMS), and it can be thought of as generalization of the normal forward Euler method (Kloeden et al. 1997). The stochastic numerical experiments presented here can be divided into two separate implementations. Both these codes require the stochastic integration of the $J$ Eq. (4), which we perform numerically using an EMS. Averaged quantities are calculated over many realizations of the strain statistics and initial conditions. An ensemble of particles is chosen as the initial condition, randomly distributed on a unit sphere in wavenumber space $|\boldsymbol{q}|=1$ (corresponding to an isotropic distribution in real space). Each particle is subjected to its own realization of the strain matrix, and its wavevector evolves according to Eq. (5).

\subsection{Code 1}

Using the integration process described above for the evolution matrix $J$, Code $1(\mathrm{C} 1)$ determines how the magnetic field evolves according to Eq. (7). As each particle is subjected to its own realization of the strain matrix the integral (7) over initial conditions simplifies to just the value of the integrand. Higher order quantities, such as $B^{4} \ldots$, can then be calculated before performing the final averaging over strain statistics. The initial magnetic noise is determined by (6) with $L=1$. In the case of $\kappa \neq 0$ one must also determine the value of the matrix $\Lambda(t)$ from Eq. (8). This is achieved via a forward Euler scheme.

$\Lambda_{j k}\left(t_{n+1}\right)=\Lambda_{j k}\left(t_{n}\right)+\Delta t\left(\sum_{l=1}^{3} J_{j l}^{-1}\left(t_{n}\right) J_{k l}^{-T}\left(t_{n}\right)\right)$.

We see therefore, that we must also determine the value of $J^{-1}$. This can be done in two different ways. Firstly, we could calculate the inverse of $J$ at each step in the usual manner by finding the adjoint and determinant of $J$. However, as some elements of $J$ can become very large in time one needs to be careful in finding the determinant as round-off errors can be easily introduced. Alternatively, we can integrate the separate stochastic equation for the evolution of $J^{-1}$

$\frac{\mathrm{d}}{\mathrm{d} t}\left(J^{-1}\right)=-\left(J^{-1}\right) \sigma$

using an EMS.

The primary aim of this code is to verify the scaling laws for the even moments $\left\langle\boldsymbol{B}^{2 n}(t)\right\rangle$, (17) and (18). However, by recording the values of the integrand of (7) and the corresponding wavenumbers $k=|\boldsymbol{k}|$ for each particle, we can also construct snapshots of the energy spectrum in time.

$\mathrm{C} 1$ has also been set up to calculate the eigenvalues of the matrix $J J^{T}$ so that the Lyapunov exponents can be calculated. Indeed, one important result for systems with random time-dependent strain is that, for nearly all realizations of the strain, the matrix $(1 / t) \ln \left(J^{T} J\right)$ is found to stabilize at large time (Goldhirsch et al. 1987; Falkovich et al. 2001). The eigenvectors $\boldsymbol{f}_{i}$ in this case tend to a fixed orthonormal basis for each realization, where $i=1,2,3$. As the Jacobian $J$ at $t=0$ is the identity matrix, $J^{T} J$ will also take the form of the identity initially. In time the strain $\sigma$ will distort $J$ via (4). An initial sphere will be deformed into an ellipsoid, the volume of which will be conserved by the incompressibility condition $\operatorname{Det}[J]=1$. The length of the principal axis of the ellipsoid correspond to the eigenvalues $\mathrm{e}^{2 \rho_{i}}$ of $J^{T} J$, while the eigenvectors $\boldsymbol{f}_{i}$ give the directions of these axis. It is evident that in considering a timedependent stochastic strain we have moved to a system corresponding to Lagrangian chaos. In which case, we can define the Lyapunov exponents $\lambda_{i}$ in terms of the limiting eigenvalues (Goldhirsch et al. 1987; Soward 1994; Falkovich et al. 2001) as

$\lambda_{i}=\lim _{t \rightarrow \infty} \frac{1}{2 t} \ln \left(\boldsymbol{f}_{i}^{T}\left(J^{T} J\right) \boldsymbol{f}_{i}\right)$,

for $i=1,2,3$. It is customary to order the Lyapunov exponents in terms of size, $\lambda_{1}>\lambda_{2}>\lambda_{3}$. A material element aligned along one of the eigenvectors $f_{i}$ will in the asymptotic limit expand or contract at the rate $\exp \left(\lambda_{i} t\right)$. The Lyapunov exponents do not depend on the realizations of the strain, while in contrast the asymptotic eigenvectors are realization dependent. Incompressibility ensures that $\sum_{i=1}^{3} \lambda_{i}=0$ which in turn tells us that one Lyapunov exponent must be positive if two or more are non-zero. A positive Lyapunov exponent corresponds to exponential growth of a material line element.

In the non-degenerate case, if the statistics of the strain matrix are symmetric to time reversal, which is true if the strain is assumed delta correlated as is the case for the KKM, then $\lambda_{2}=0$ (Kraichnan 1974; Balkovsky \& Fouxon 1999) and hence the incompressibility condition gives $\lambda_{3}=-\lambda_{1}<0$. Chertkov et al. (1999) found that it is the largest Lyapunov exponent $\lambda_{1}$ that is responsible for the growth of the moments $<\boldsymbol{B}^{2 n}(t)>$. Balkovsky \& Fouxon (1999) have also considered the matrix $J J^{T}$, which evolves in time according to

$\frac{\mathrm{d}}{\mathrm{d} t}\left(J J^{T}\right)=\sigma\left(J J^{T}\right)+\left(J J^{T}\right) \sigma^{T}$

This differs from the matrix $J^{T} J$ in that it does not stabilize to some fixed axis at large time. Indeed, the eigenvectors will 

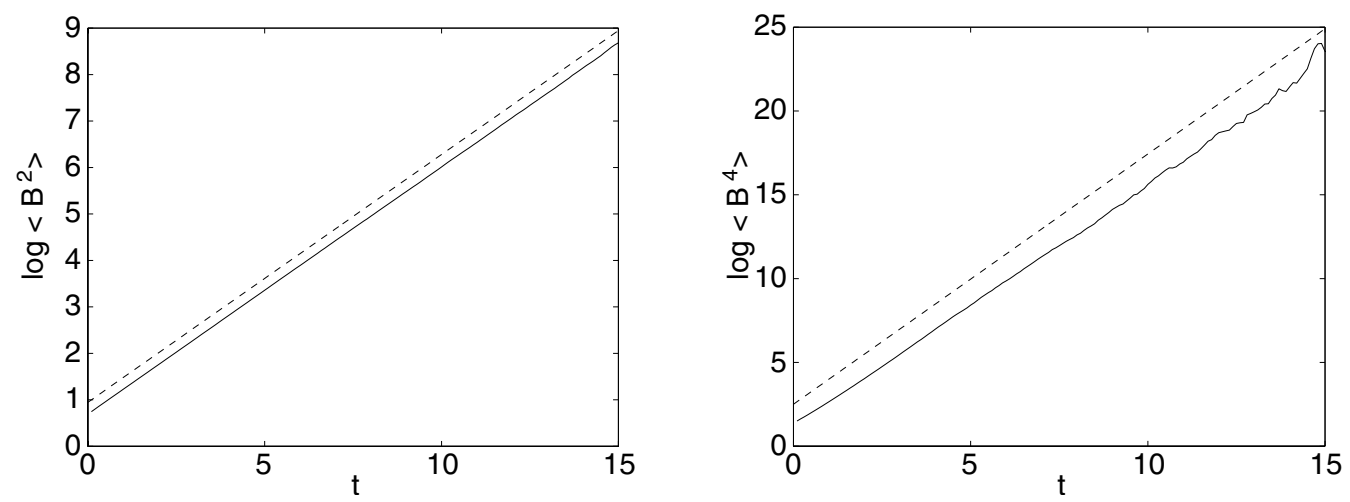

Fig. 1. $\log <\boldsymbol{B}^{2}(t)>$ (left figure) and $\log <\boldsymbol{B}^{4}(t)>$ (right figure) for a $\mathrm{C} 1$ simulation, with $\Omega=0.16$ and $\kappa=0$. Averaging was performed over 600000 realizations. The theoretical solutions (17) have also been plotted (dashed lines).

continue to rotate for every realization of the strain matrix. As in (33), the Lyapunov exponents can also be found from the logarithm of the eigenvalues of $J J^{T}$ at large time. The eigenvalues of $J J^{T}$ and $J^{T} J$, and hence their Lyapunov exponents, are the same as they share the same characteristic polynomial.

To determine the Lyapunov exponents of our system numerically we need to determine either $J J^{T}$ or $J^{T} J$ at each timestep. This can be achieved by either calculating $J J^{T}$ via its own dynamic Eq. (34) and integrate it using an EMS, or alternatively calculate $J J^{T}$ or $J^{T} J$ at each timestep from $J$. In practice either approach works equally well. Next one must determine the eigenvalues of the symmetric matrix $J J^{T}$ which must be real. Although the matrix is only $3 \times 3$ solving the characteristic polynomial using the solution to a cubic equation is impractical as the growth/reduction of elements $J$ in time produce round-off errors numerically. This in turn leads to complex eigenvalues and further inaccuracies.

The method employed in this numerical study is that of Jacobi transformations (Press et al. 1993). The key to this method is a sequence of similarity transformations (rotations). In the context of ellipsoids, this algorithm can be thought of as a method by which the axis of the system are rotated and realigned to lie along the principle axis of the ellipsoid, the length of these axis corresponding to the eigenvalues, and the directions to the eigenvectors. Although the Jacobi rotation method is not perfect, it does however perform better than the previously mentioned cubic equation approach, in that the eigenvalues remain real for all time. Given the set of eigenvalues $\exp \left(2 \rho_{i}\right)$ of $J J^{T}$, we define the time-dependent Lyapunov exponents to be

$\lambda_{i}(t)=\frac{1}{2 t}<\ln \left(\exp \left(2 \rho_{i}\right)\right)>=\frac{1}{t}<\rho_{i}>$,

where $i=1,2,3$. Analytically the Lyapunov exponents are independent of the strain realizations at large time, but the averaging above has been performed over all the realizations and initial conditions to improve accuracy.

\subsection{1. $\kappa=0$ simulations}

We will start by setting $\kappa=0$ in the numerics so that we can investigate the perfect conductor regime. Firstly, let us compare the scaling of $\left\langle\boldsymbol{B}^{2}(t)>\right.$ with theoretical solution (17). The left-hand graph of Fig. 1 corresponds to the $n=1$ case and we see the numerically generated slope agrees nicely with the theoretical scaling (dashed line). For the higher order moments $<\boldsymbol{B}^{2 n}(t)>$, good agreement with the analytical scaling (17) can also be seen. The right-hand graph in Fig. 1 shows the slope for $n=2$. As all moments are calculated from the integral (7), it should be noted that any fluctuations in $\boldsymbol{B}^{2}(t)$ will get amplified at higher orders, consequently the resulting graphs will become progressively noisier. The only solution to this problem is to increase the number of the realizations over which averaging is performed.

Let us now investigate the energy distribution in wavenumber space. The energy spectrum $\left\langle\boldsymbol{B}^{2}(k, t)\right\rangle$ is obtained at a particular instant in time by recording each particles wavenumber $k=|\boldsymbol{k}|$ and "weight" corresponding to the integrand of (7). One then constructs a histogram by first finding the largest and smallest wavenumbers $k_{\max }$ and $k_{\min }$ of the all the particles, and then dividing the interval $\log k_{\min }<\log k<\log k_{\max }$ into a finite number of bins. Particles are then sorted into these bins and their corresponding weights are summed. Finally, we normalize the total weight in each bin, by dividing through by its bin width. One must also divide through by a factor of $4 \pi k^{2}$ which originates from the solid angle integration required in converting a 1D spectrum into that of a 3D one (see for example McComb 1990).

In Fig. 2 the energy spectrum has been plotted for two different times $t=9$ (left figure) and $t=18$ (right figure). Here $\Omega=0.16$ and thus using the definition (15) for the critical wavenumber $k_{c+}(t)$ we obtain $\log _{10} k_{c \pm}(9)= \pm 0.6$ and $\log _{10} k_{c \pm}(18)= \pm 0.85$. In Fig. 3 the same spectrum has been plotted for $t=27$ (left figure) and $t=31.5$ (right figure), correspondingly $\log _{10} k_{c+}(27)=1.04$ and $\log _{10} k_{c+}(31.5)=1.13$. One should recall that theoretically for wavenumbers $k_{c-} \ll$ $k \ll k_{c+}$ we expect a $k^{-1 / 2}$ scaling range ${ }^{3}$, and this is indeed the case here (the straight line in each graph corresponding to a slope of $k^{-1 / 2}$ ). As a whole we can see that the spectrum moves vertically upwards in time. This corresponds to the time dependent increase of the magnetic energy at fixed $k$ predicted in the

\footnotetext{
${ }^{3}$ Here, we are considering a 3D spectrum. Kulsrud \& Anderson (1992) considered the equivalent 1D spectrum which has a corresponding $k^{3 / 2}$ scaling regime.
} 

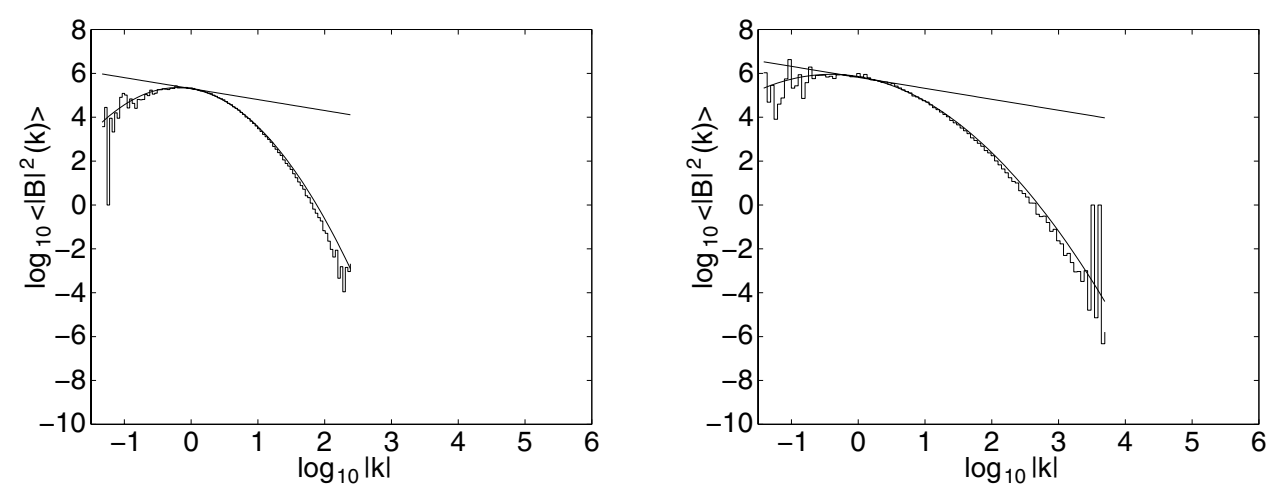

Fig. 2. $E=\left\langle\boldsymbol{B}^{2}(k, t)>\right.$ for a $\mathrm{C} 1$ simulation with $\Omega=0.16$ and $\kappa=0$. Averaging has been performed over 600000 realizations. The time is $t=9$ (left figure) and $t=18$ (right figure). The particles have been sorted into 100 bins. The theoretical solution (14) (curve) and a slope of $k^{-1 / 2}$ (straight line) have also been plotted.
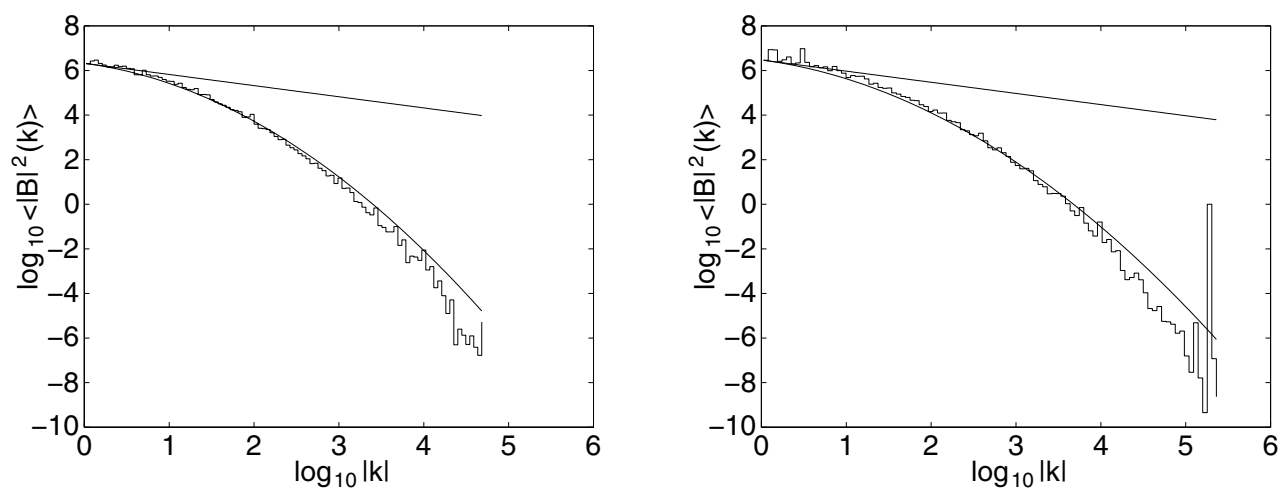

Fig. 3. $E=\left\langle\boldsymbol{B}^{2}(k, t)>\right.$ for a $\mathrm{C} 1$ simulation with $\Omega=0.16$ and $\kappa=0$. Averaging has been performed over 600000 realizations. The time is $t=27$ (left figure) and $t=31.5$ (right figure). The particles have been sorted into 100 bins. The theoretical solution (14) (curve) and a slope of $k^{-1 / 2}$ (straight line) have also been plotted.

theoretical result (14). This large time asymptotic solution has also been plotted on each figure and we see good agreement with the numerically generated histograms.

It should be noted that the later figures show the spectrum for $\log _{10} k>0$. This is because the front propagating to small $k$ becomes extremely noisy at large time. The reason for this will become apparent shortly. It is evident from these results that it would be beneficial if we could extend the number of decades over which this scaling is observed. A possible approach would be to run the simulations for a much longer time, remembering that $\log k_{c \pm}(t) \sim \sqrt{\Omega t}$. In doing this one has to be careful of numerical stability and the generation of round-off errors as some elements of $J$ will get ever larger. However, this is not the only problem, we should remember that our distribution of particles, initially concentrated at our initial wavenumber $|\boldsymbol{q}|=1$, will broaden in time. The peak of this distribution will move towards larger $k$, each individual particle performing its own random walk in wavenumber space. Figure 4 shows the distribution of particles at four successive times $t=9,18,27$ and 35 for a simulation with $\Omega=0.16$. The graph has been normalized so that the area under the curve is one. We see that with log wavenumber coordinate, the particle distribution fits a moving Gaussian profile. The mean of this profile moves to higher wavenumbers as $\Omega t$, while the standard deviation grows as $\sqrt{\Omega t}$. Regrettably, the energy spectrum's $k^{-1 / 2}$ scaling range of interest lies in one of the tails of the particle distribution.
This means that there will be fewer particles available for averaging when calculating the energy spectrum histogram in this region.

The only way to improve this averaging is to increase the total number of particles used in a simulation. Of course, this is not an efficient way of improving the statistics, as increasing the number of rarer particles located in each tail, will correspond to a much greater increase in the number of particles located in the center of the particle distribution. This is an inherent problem with this type of particle simulation. Also, as the particle distribution gets more spread out in time, fluctuations at both ends of the energy spectrum histogram will increase. This effect can clearly be seen in Figs. 2 and 3. This is why the front $k_{c-}$ that propagates to smaller $k<q<1$ in time becomes so noisy, as the bulk of the particles are traveling to larger wavenumbers. For this reason in the remaining results we will concentrate only on the $\log _{10} k \geq 0$ region of $k$-space.

\subsection{2. $\kappa \neq 0$ simulations}

We will now introduce a non-zero $\kappa$ into the numerical model to investigate the effects of diffusion. The first thing we will investigate here is the scaling behavior of the total magnetic energy $\left\langle\boldsymbol{B}^{2}(t)>\right.$ in the diffusive regime. The left-hand graph of Fig. 5 shows the results of a simulation with $\Omega=0.36$ and $\kappa=0.005$. The two separate scaling regimes are clearly 

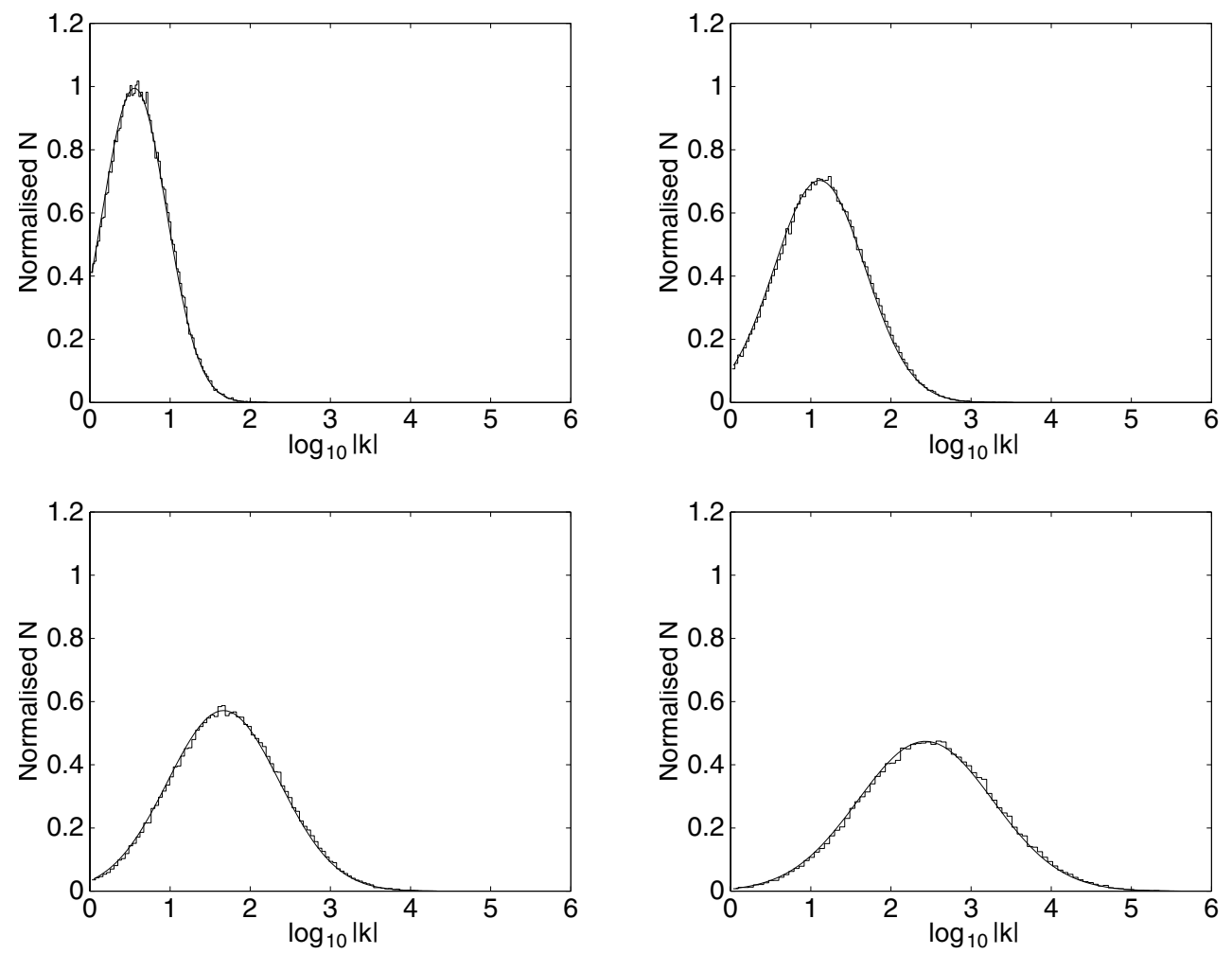

Fig. 4. Normalized particle distribution $N(k, t)$ for a $\mathrm{C} 1$ simulation with $\Omega=0.16$. Averaging has been performed over 120000 realizations. Snapshots are shown at $t=9$ (top left), $t=18$ (top right), $t=27$ (bottom left) and $t=35$ (bottom right). The particles have been sorted into 100 bins. A Gaussian curve has also been fitted to the distribution.
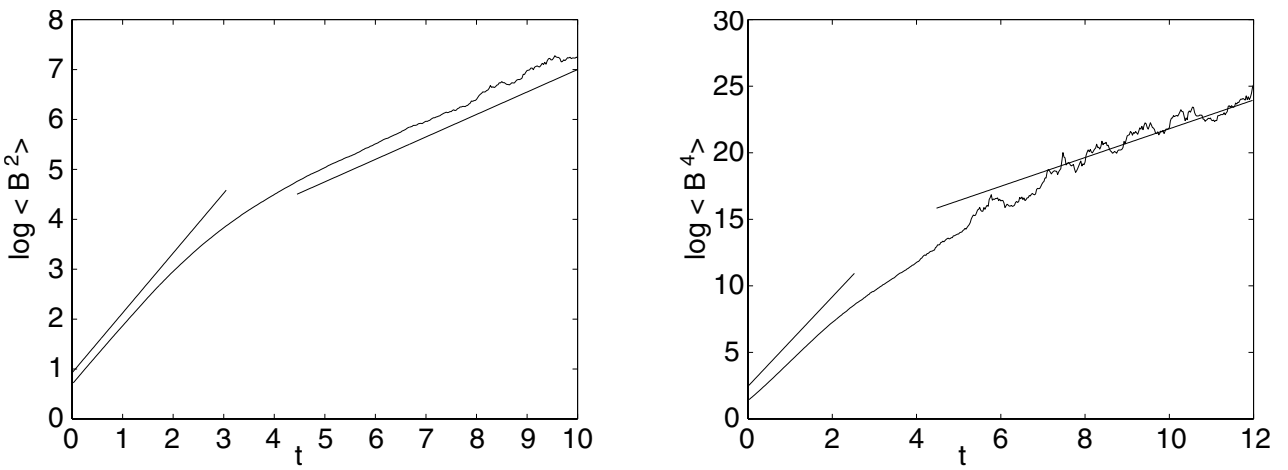

Fig. 5. $\log \left\langle\boldsymbol{B}^{2}(t)\right\rangle$ (left figure) and $\log \left\langle\boldsymbol{B}^{4}(t)\right\rangle$ (right figure) for a $\mathrm{C} 1$ simulation, with $\Omega=0.36, \kappa=0.005$. Averaging was performed over 120000 realizations (left figure) and 480000 realizations (right figure). The theoretical slopes (17) and (18) have also been plotted (dashed lines).

apparent and the analytical slopes (17) and (18), for the perfect conductor and diffusive regimes respectively, are in good agreement with the numerics. It is inevitable that to generate similarly smooth graphs for the higher order moments, one would have to use a greater number of realizations. Figure 6 and the right-hand graph of Fig. 5 show the scaling of the next three even moments. As expected these graphs are noisier, but still in agreement with the analytical results.

Next we will consider the energy spectrum. The introduction of a finite diffusivity will cut-off the energy spectrum at some finite wavenumber. Figure 7 shows two successive snapshots of the energy spectrum at times $t=6$ and 12 for a simulation with $\kappa=0.005$ and $\Omega=0.36$. As expected, one observes a spectral cut-off and for the results shown in Fig. 7, the parameter choice gives $k_{\kappa}=\sqrt{\Omega / 6 \kappa}$. Theory predicts that for $k<k_{k}$ there should be a region of $k^{-1 / 2}$ scaling (19) (with a logarithmic correction). For this particular simulation we have $\log _{10} k_{k} \simeq 1.1$. As with the perfect conductor regime, the spectrum is seen to move vertically upwards corresponding to a steady increase in the total magnetic energy. The large time theoretical solution (19) has also been plotted in Fig. 7 and good agreement is observed.

We will briefly review the Lyapunov exponents generated in these $\mathrm{C} 1$ numerical experiments because it help us to understand the origins of some numerical errors in our method. Using the Jacobi transform method described earlier, one can calculate the eigenvalues $\mathrm{e}^{2 \rho_{i}}$ from either $J^{T} J$ or $J J^{T}$, and then order them in terms of size so that $\rho_{1}>\rho_{2}>\rho_{3}$. 

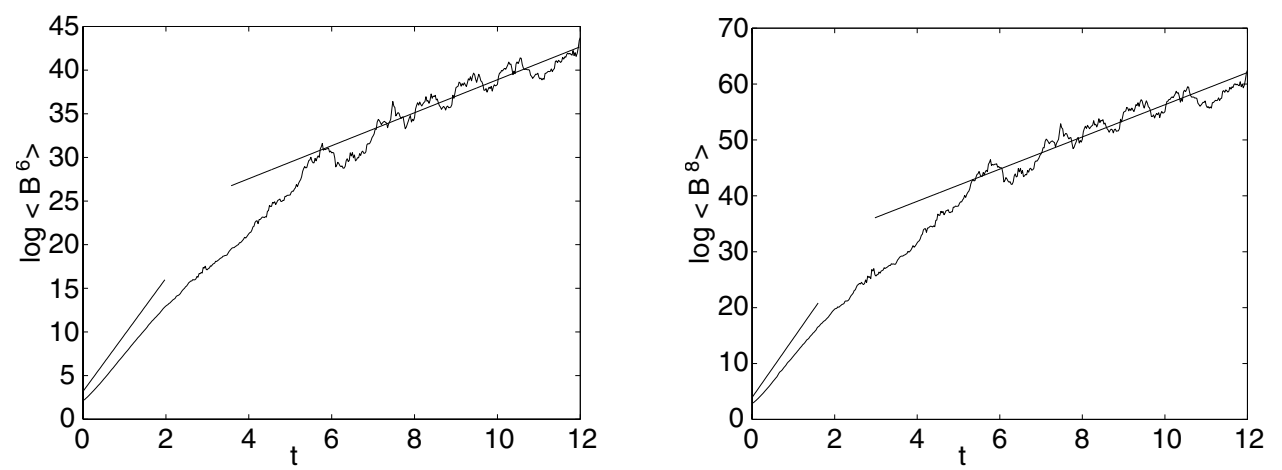

Fig. 6. $\log \left\langle\boldsymbol{B}^{6}(t)>\right.$ (left figure) and $\log \left\langle\boldsymbol{B}^{8}(t)>\right.$ (right figure) for a C1 simulation with $\Omega=0.36$ and $\kappa=0.005$. Averaging has been performed over 480000 realizations. The theoretical slopes (17) and (18) have also been plotted (straight lines).
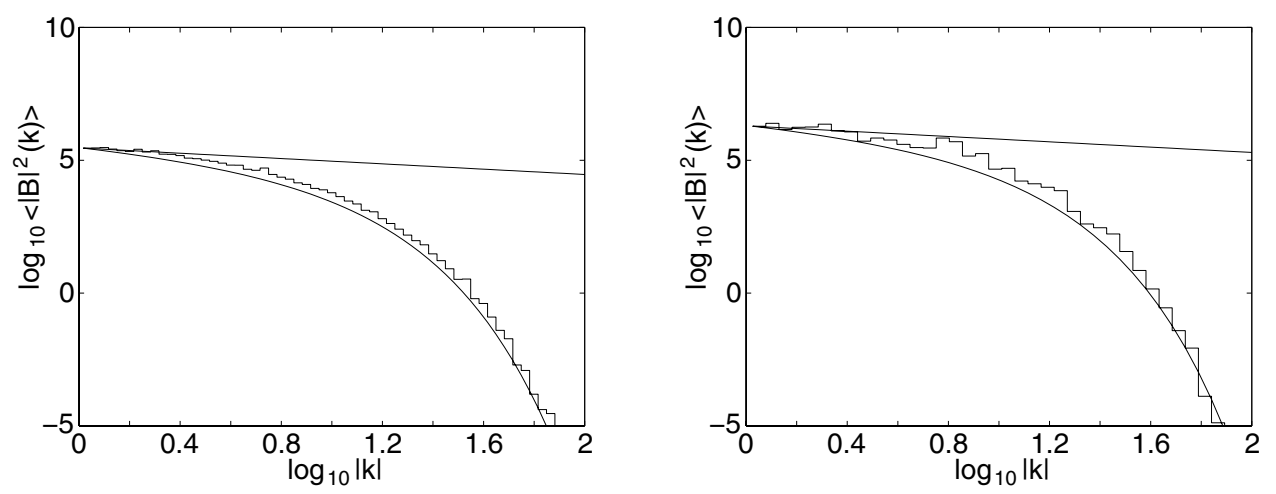

Fig. 7. $E=\left\langle\boldsymbol{B}^{2}(k, t)>\right.$ for a $\mathrm{C} 1$ simulation with $\Omega=0.36$ and $\kappa=0.005$. Averaging has been performed over 480000 realizations. The time here is $t=6$ (left figure) and $t=12$ (right figure). The particles have been sorted into 100 bins. The theoretical solution (19) (curve) and a slope of $k^{-1 / 2}$ (straight line) have also been plotted.

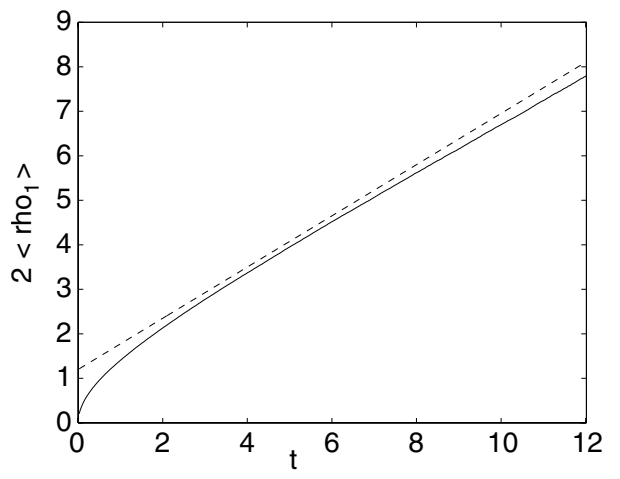

Fig. 8. $<2 \rho_{1}(t)>$ for a $\mathrm{C} 1$ simulation with $\Omega=0.36$. Averaging has been performed over 480000 realizations. A slope of $(8 / 5) \Omega t$ has also been plotted (dashed line).

The Lyapunov exponents are then determined via (35). Figure 8 shows $<2 \rho_{1}(t)>$ for a simulation with $\Omega=0.36$. As expected the graph tends to a flat line in time, and $\lambda_{1}>0$. We would expect $\lambda_{1} \sim \Omega$, that is $\left\langle\ln \mathrm{e}^{2 \rho_{1}}>=2 t \lambda_{1}=2 t C \Omega\right.$ where $C$ is the time-scaling constant mentioned earlier. In Fig. 8 a line corresponding to $C=4 / 5$ has also been plotted. From theory we would expect the remaining two Lyapunov exponents to take the values $\lambda_{2}=0$ and $\lambda_{3}=-\lambda_{1}$, with conservation of volume corresponding to $\sum \lambda_{i}=0$. The left and right-hand graphs of Fig. 9 shows $<2 \rho_{2}(t)>$ and $<2 \rho_{3}(t)>$ respectively. Looking at the range of the vertical axis we see $\lambda_{2}$ does indeed stay around zero but there is a gradual deviation from the theoretical value in time. On the other hand, $\lambda_{3}$ is not the same as $-\lambda_{1}$. The line $<\ln \mathrm{e}^{2 \rho_{3}}>=-2 t \lambda_{1}=-2 t C \Omega$ with $C=4 / 5$ has also been plotted for comparison. Numerically, we see that $\sum \lambda_{i} \neq 0$ and hence, according to the Lyapunov exponents, the volume is not conserved. It should also be mentioned that in our simulations, the determinant of the Jacobian $\operatorname{Det}[J]$, is held within $1 \%$ of unity, which means that the volume is well conserved. We conclude therefore that the deviations observed in the numerical values of $\lambda_{i}$ from theory are mainly due to errors generated in finding the eigenvalues of $J J^{T}$ or $J^{T} J$ using the Jacobi transform method. This is not overly surprising if we remember that some elements of $J J^{T}$ are very large in comparison to others. Indeed, this is why the alternative method of finding the eigenvalues via solving the cubic characteristic polynomial of $J J^{T}$ runs into difficulties. In the context of the ellipsoids, essentially the problem arises because each ellipsoid is becoming ever larger in one direction and smaller in another.

It should be noted that the magnetic Reynolds number is relatively small in these simulations. The magnetic Reynolds number is by definition $R_{\mathrm{m}}=\sqrt{L^{2} \Omega / \kappa}$, giving a range of values 32 to 72 in the above simulations, where we have taken $L$ to be of unity. Thus, it is only the relative sizes of $\Omega$ and $\kappa$ that are important. Higher Reynolds number simulations are possible but again increasing $\Omega$ will make the stochastic fluctuations greater and hence one will need to reduce the timestep in each simulation. Alternatively, decreasing the diffusivity $\kappa$ will delay 

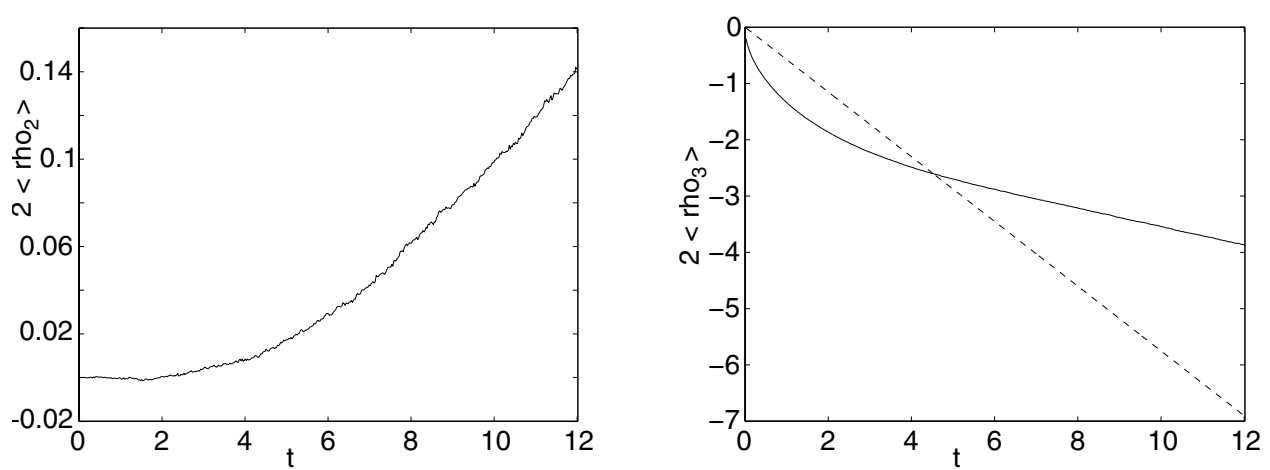

Fig. 9. $<2 \rho_{2}(t)>$ (left figure) and $<2 \rho_{3}(t)>$ (right figure) for a $\mathrm{C} 1$ simulation with $\Omega=0.36$. Averaging has been performed over 480000 realizations. A slope of $-(8 / 5) \Omega t$ (right arrow) has also been plotted (dashed line).
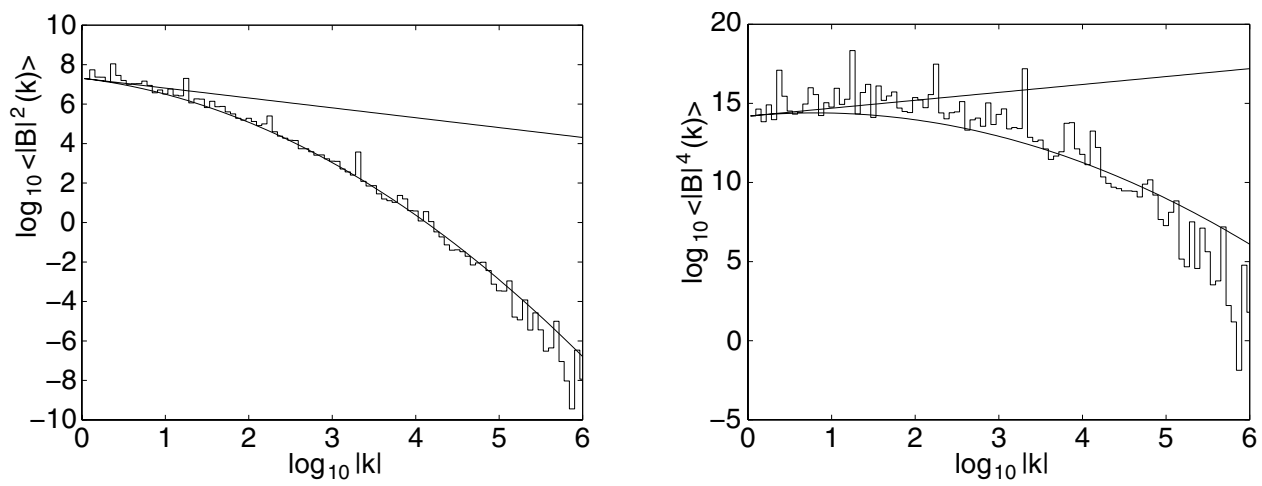

Fig. 10. $E=\left\langle\boldsymbol{B}^{2}(k, t)\right\rangle$ (left figure) and $S=\left\langle|\boldsymbol{B}|^{4}(k, t)\right\rangle$ (left figure) for a C2 simulation with $\Omega=0.16$ and $\kappa=0$. The time here is $t=35$. Averaging has been performed over 600000 realizations. The particles have been sorted into 100 bins. The theoretical solution (14) (curve) and a slope of $k^{-1 / 2}$ (straight line) have also been plotted in the left figure. While the curve (22) and a slope of $k^{+1 / 2}$ (straight line) have also been plotted in the right figure.

the time at which transition to the dissipative regime occurs. In this case, the corresponding simulation will have to be run over a larger time interval and more particles would have to be used to fill the enlarged wavenumber volume in order to achieve good statistics. Note that the detrimental effect of insufficient number of particles is most felt in the low-wavenumber part of the spectrum due to a fewer number of particles in the spherical shells. This situation is common for all simulations discussed in the present paper and the maximal possible magnetic Reynolds number will be dictated by the available computer resources.

\subsection{Code 2}

Code 2 (C2) uses the same foundations as $C 1$, an ensemble of particles are initially distributed on the surface of a unit sphere in wavenumber space. However, now each particle is given a randomly orientated magnetic field satisfying $\boldsymbol{B} \cdot \boldsymbol{q}=0$. That is, the real and imaginary parts of the magnetic field are randomly orientated in a plane which is at right-angles to the particle's initial wavevector and tangent to the unit sphere in $k$-space. Particles are again allowed to evolve in wavenumber space via (5), but in contrast to $\mathrm{C} 1$, information about the full magnetic field is retained by the integration of Eq. (3). In the case of $\kappa \neq 0$ we need to determine the integral in (3). As with $\mathrm{C} 1$ this is achieved through the use of a forward Euler scheme. In this way one can investigate the spectra of Fourier space quantities of interest such as the magnetic energy, polarization and flatness.

\subsection{1. $\kappa=0$ simulations}

Starting with the energy spectrum $\left\langle\boldsymbol{B}^{2}(k, t)>\right.$. This has been plotted in the left-hand graph of Fig. 10 along with the large time theoretical curve (14) and slope $k^{-1 / 2}$, for the choice of parameters $\Omega=0.16$ and $t=35$. As with the $\mathrm{C} 1$ experiment Fig. 3, the numerical and analytical results support each other well. As with the investigation of higher order moments $<\boldsymbol{B}^{2}(t)>$ in Sect. 4.1.1, the higher order correlators, such as $S=\left\langle|\boldsymbol{B}|^{4}(k, t)>\right.$, will generate noisier histograms in comparison to their lower order counterparts. Nevertheless, the following numerical results are still very much of interest. Firstly, we will examine the correlator $S$ which has been plotted as the right-hand graph of Fig. 10. The large time asymptotic solutions has also been plotted, and there appears to be good qualitative agreement with the numerics, although some deviation is evident at large $k$. As with the energy spectrum the analytical results tell us that there exists a $k^{+1 / 2}$ scaling range for $k<k_{c+}$. In this simulation $\log _{10} k_{c+}=1.13$. A slope of $k^{+1 / 2}$ has been included in the graph and again there would seem to be agreement. However, it must be said that the $k$-interval for this scaling range is perhaps too short and the histogram too noisy to be conclusive. 

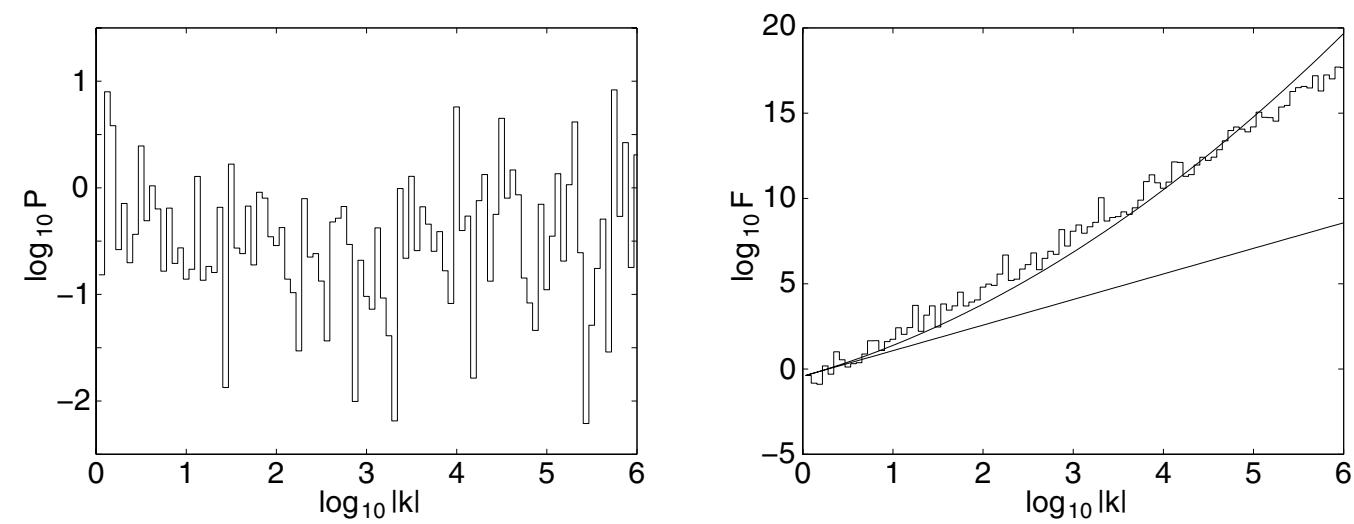

Fig. 11. $P=(S-T) / S$ (left figure) and $F=S / E^{2}$ (right figure) for a $\mathrm{C} 2$ simulation with $\Omega=0.16$ and $\kappa=0$. The time here is $t=35$. Averaging has been performed over 600000 realizations. The particles have been sorted into 100 bins. In the right figure the theoretical curve (27) and slope $k^{+3 / 2}$ (straight line) has also been plotted.

For the same simulation, Fig. 11 shows the normalized mean polarization $P$ (left figure) and spectral flatness $F$ (right figure). Let us consider the $P$ spectrum. From the theoretical results we expect $P$ to be independent of wavenumber. Although, the histogram is noisy, there does indeed appear to be no $k$-dependence, and the resulting spectrum is flat. However, for the KKM we also have the prediction that the polarization tends to zero in time (25). Unfortunately, this is not the case numerically. Indeed, taking an average over all $k$ in the histogram we can find an approximate value for $P$ from the above spectrum. Repeating this procedure for various snapshots in time we find the normalized polarization remains steady in time at the value of 0.32 . One should remember that for a Gaussian field analytically we found $P=1 / 3$. Therefore, in our numerical simulation the polarization seems to be that of a Gaussian field. However, the corresponding spectral flatness is far from being Gaussian. Indeed, the flatness $F$ in this perfect conductor simulation can be seen in the right-hand graph of Fig. 11. Below $\log _{10} k_{c+}=1.13$ we see agreement with the analytical solution of $k^{+3 / 2}$. While at larger $k$ the theoretical increase in the flatness, due to the log squared term in (27), is also well represented.

One may well ask, why is there a discrepancy between theory and the numerically generated polarization, but not the flatness? It helps if we can understand the physical significance of these two quantities. We return to the familiar example of the evolution of an initial ball of isotropic magnetic turbulence is wavenumber space. For each realization this ball is deformed into an ellipsoid with one large, one short and one neutral direction. One can visualize this ellipsoid as an elongated flat cactus leaf with thorns aligned to the magnetic field direction. Note that in this picture one component of the magnetic field (transverse to the cactus leaf) is dominant which is captured by the fact that the polarization $P$ introduced above, tends to zero at large time. Another consequence of this picture is that the wavenumber space will be covered by the ellipsoids more sparsely at large $k$. This produces large intermittent fluctuations of the Fourier transformed magnetic field. These fluctuations are quantified by the growth of the flatness $F$ as $k^{3 / 2}$, a clear indication of this small-scale intermittency.
Numerically, it would appear that the flatness is a little more robust than the polarization, arising naturally in the numerics as the ellipsoids become increasingly sparse at large $k$. In contrast, the apparently Gaussianity of the polarization can be attributed to the mis-alignment of the cactus needles. Indeed, the magnetic needles should align themselves with the smallest principal axis of the ellipsoid. In this direction the cactus leaf is becoming continuously thinner in time, and hence numerically one might expect some errors to creep into the alignment of $\boldsymbol{B}$. Indeed, this seems to be related to the errors observed in calculating the smallest Lyapunov $\lambda_{3}$.

In fact, although on average our numerical simulations appear to mis-represent the behavior of the polarization completely, things are not as bad as they would initially seem. Indeed, further insight can be obtained by considering the behavior of an ensemble of particles that are subjected to the same realization of the strain. Figure 12 shows the real magnetic fields of a set of 500 wavepackets that have been subjected to two different realizations of the strain matrix ${ }^{4}$. In the left-hand figure it is clear the magnetic field in this realization is far from being plane polarized, the magnetic vectors still being predominantly random in their orientation at this given point in time $t=6$. In contrast, in the right-hand figure, which has also been taken at $t=6$, we see the ellipsoid has become very elongated and the magnetic field appears plane polarized. Earlier snapshots, at $t=2$ and 5, of the magnetic field for this particular realization can be found in Fig. 13, and the evolution of the corresponding polarization spectra is shown in Fig. 14. In this case, we see the polarization does indeed decay in time and is far from being Gaussian by $t=6$. In some respects, therefore, our numerical model does get some of the polarization's behavior at least qualitatively correct.

Before we consider the effects of finite diffusivity, we should check how the growth rates of the spectra behave in time. That is, how rapidly they move vertically up or down in time. By recording the value of each spectra for $k=1$, at different snapshots in time, we can compare this numerical growth to the theoretical solutions (14), (22), (23) and (27) for $E, S, T$

\footnotetext{
4 The imaginary magnetic fields are qualitatively similar and have therefore not been included.
} 

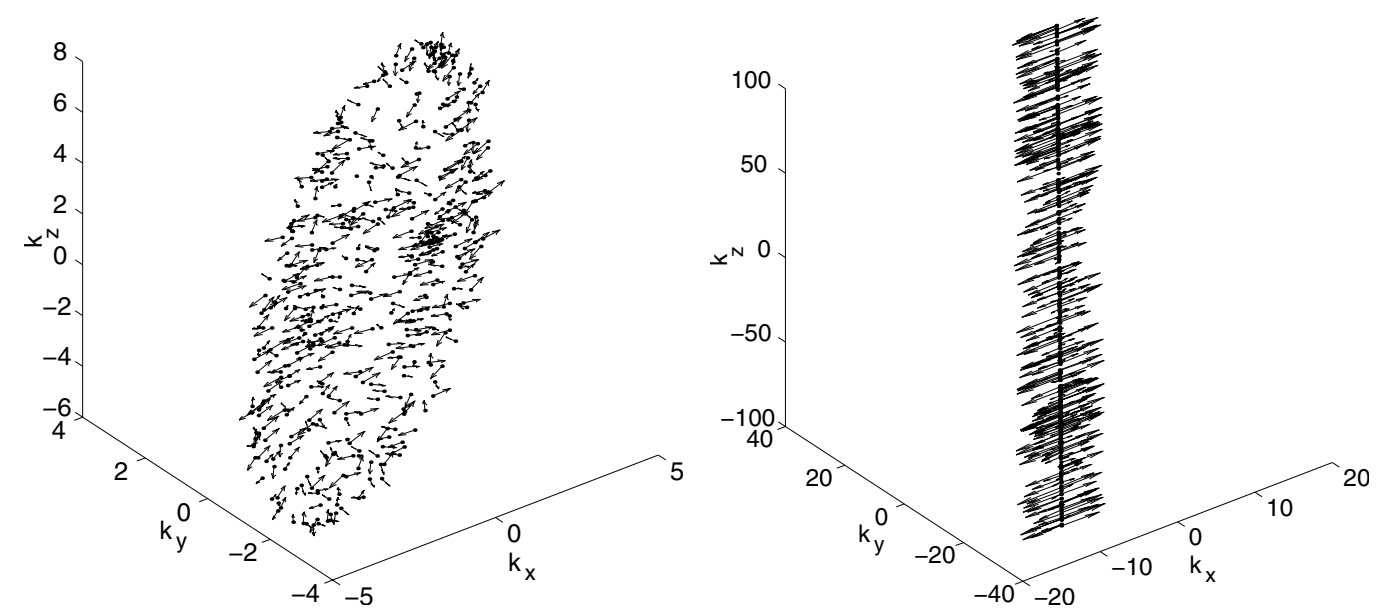

Fig. 12. The magnetic field of 500 wavepackets in a $C 2$ simulation with $\Omega=0.36$ and $\kappa=0$. The figures show the particle's positions in $k$-space at $t=6$ and their corresponding real magnetic fields. The left and right figures correspond to different realizations of the strain matrix.
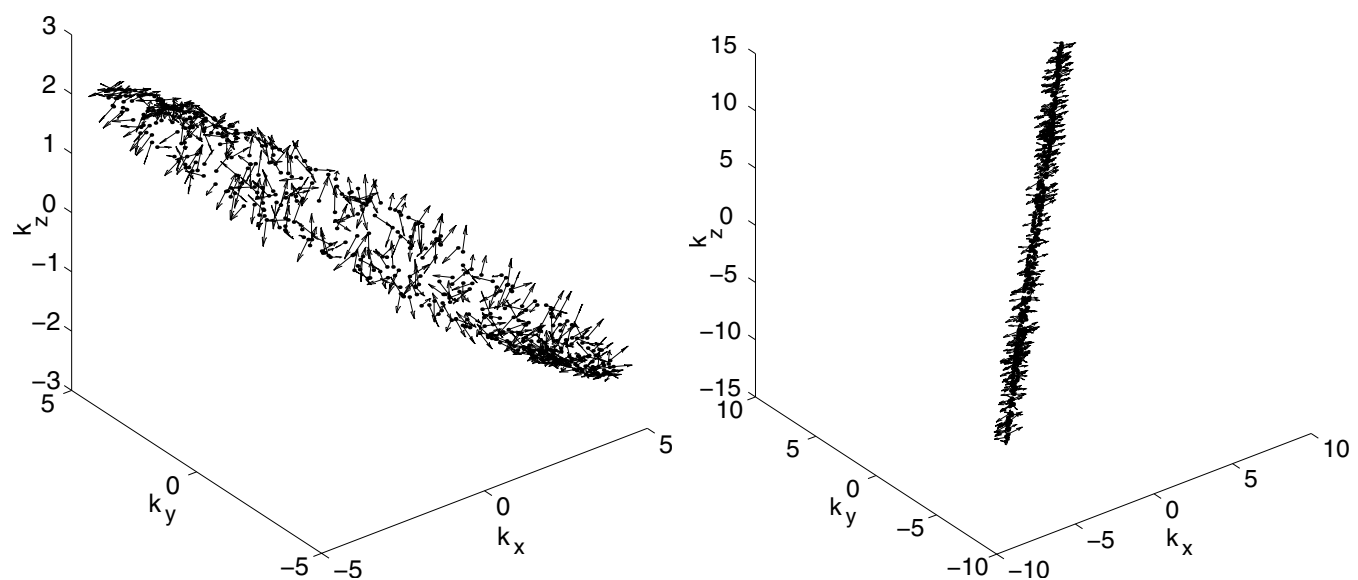

Fig. 13. The magnetic field of 500 wavepackets in a $C 2$ simulation with $\Omega=0.36$ and $\kappa=0$. The figures show the particle's positions in $k$-space and their corresponding real magnetic fields at $t=2$ (left figure) and $t=5$ (right figure) for one realization of the strain matrix. The strain field is the same here as the realization used in right-hand graph Fig. 12.

and $F$ respectively. These results can be found in Fig. 15. Along with the numerical values (points) the corresponding theoretical growth has also been plotted (solid line). It should be noted that this is only a rough check. Firstly, because we have had to ignore the $\sqrt{t}$ prefactor in each analytic solution, and secondly because the numerical values of the spectra at $k=1$ are likely to fluctuate at large time as we are again in the tails of the particle distribution. Nevertheless, all the spectra are growing in time in agreement with theory.

\subsection{2. $\kappa \neq 0$ simulations}

In this section we will discuss the results of a set of $\mathrm{C} 2$ simulations with a finite diffusivity of $\kappa=0.005$. Figure 16 shows snapshots of $\left\langle\boldsymbol{B}^{2}(\boldsymbol{k}, t)\right\rangle$ at the times $t=6$ and 12. As with the $\mathrm{C} 1$ simulations, the finite diffusivity produces a spectral cut-off. As expected the spectrum moves vertically upwards in time, with the second histogram getting noisier as the density of particles in the interval $k<k_{c+}$ is reduced. Comparing these energy spectra to their equivalent $C 1$ counterparts, Fig. 7, we should note that the $C 2$ numerics appear to be slightly hyper-diffusive. This effect is most probably numerical, originating from the integration of the $k^{2}$ integral found in (3).

The higher order correlators $S$ and $T$ have been plotted in Fig. 17 for a time $t=6$. We should note that the particles here have been sorted into 100 bins. However, the axis in each graph has been re-scaled to take into account the spectral cutoff. For small $k$, qualitatively these figures are in agreement with the large time asymptotic solutions. However, as with the energy spectrum, these results seem to slightly hyper-diffusive. The final figure we will consider is 18 which shows flatness $F$ at $t=6$. Again we observe good qualitative agreement with theory. In particular, one can observe a $k^{3 / 2}$ scaling region for small $k$ and a heightened flatness at larger $k$. This coincides with the analytical prediction that the inclusion of a finite diffusivity increases the flatness (and hence small-scale intermittency) at large $k$. The corresponding normalized mean polarization spectrum has not been included here as it is again flat, much like the perfect conductor regime (see Fig. 11) but with a spectral cut-off. As with the non-diffusive case, on average the normalized polarization appears Gaussian with $P=0.32$. Again, it is interesting to consider the magnetic field of an ensemble of particles that have been subjected to the same 


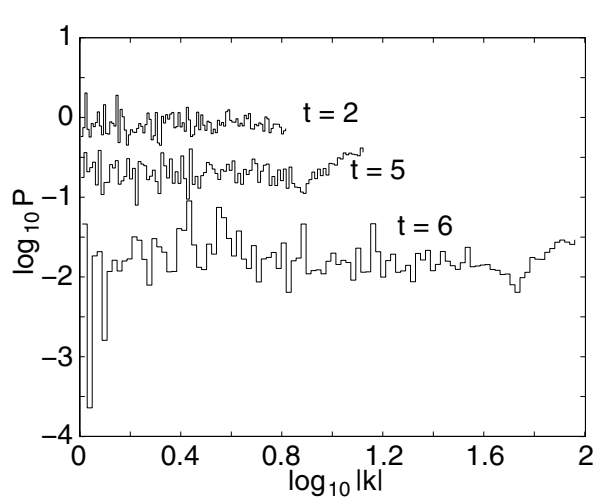

Fig. 14. The polarization $P=Q / S$ of 500 wavepackets in a $\mathrm{C} 2$ simulation with $\Omega=0.36$ and $\kappa=0$. Here all the particles are subjected to the same realization of the strain matrix. The graph shows the polarization at $t=2$ (top line), $t=5$ (middle line) and $t=6$ (bottom line). The corresponding snapshots of the particle's positions and magnetic field can be found in Figs. 13 and 12 respectively.

realization of the strain matrix. Figure 19 shows two such snapshots of an ensemble of 500 magnetic particles. These two figures should be compared with Fig. 12 which show the same realizations but without diffusion. The right-hand figure in particular demonstrates why, in the diffusive regime, an ellipsoid will cover $k$-space more sparsely due to the decay of its magnetic field at its tips. This is the reason why spectral flatness increases in the diffusive regime.

\section{Numerical investigations beyond the KKM}

The purpose of the stochastic codes outlined above was to investigate the small-scale dynamo system for the case of a Gaussian white strain field. In contrast, the following two codes were developed to test the universality of the analytical KKM results summarized in Sect. 3, when more realistic representations of the velocity field are chosen. These simulations are based on the integration of the induction Eq. (1), which when written in a Lagrangian frame of reference in $k$-space ${ }^{5}$ takes the form

$\frac{\mathrm{d}}{\mathrm{d} t} B_{m}=\sigma_{m i} B_{i}-\kappa k^{2} B_{m}$,

where $\mathrm{d} / \mathrm{d} t \equiv \partial_{t}+\dot{k}_{j} \partial_{k_{j}}$ and

$\dot{k}_{j}=-\sigma_{i j} k_{i}$.

Numerically we consider an ensemble of particles (wavepackets), whose individual magnetic fields evolve according to (36) and wavevectors according to (37).

\subsection{Code 3}

In code 3 (C3), we consider a synthetic Gaussian strain field with a finite correlation time. The rms of the different strain

\footnotetext{
5 Strictly speaking, the Fourier transforms used in deriving this equation must be taken over a box. The box size is greater than the characteristic length-scale of magnetic turbulence, but less than the characteristic length-scale of the velocity field. In this case, model (2) corresponds to ignoring the quadratic terms in the scale separation parameter. The strain here is measured along the fluid path.
}

components in this numerical experiment ranged from 2.9 to 3.6 and the correlation time was 0.02 . Thus the correlation time was about sixteen times less than the characteristic strain distortion time. If we choose a timestep which is much smaller than the correlation time, we no longer need to consider a stochastic scheme, but can instead use a more traditional second-order Runge-Kutta method to integrate (36) and (37). This comes at the price: either the run has to be shorter (and therefore cover smaller wavenumber range) or number of particles has to be much less than in the stochastic runs (with detrimental consequences for the statistics). The present experiment consisted of 2048 strain realizations with 2048 magnetic wavepackets and the timestep was chosen to be twenty times smaller than the strain correlation time. Initially, the particles are randomly distributed within a ball of radius 2 in the $k$-space. Each particle is given a randomly orientated magnetic field (with random phase) that is transverse to its initial wavevectors $\boldsymbol{q}$ and which has a random amplitude less than or equal to $2 \times 10^{-4}$.

Figure 20 shows the energy spectrum (left figure) and the growth of the total energy (right figure). At large time but before the transition to the dissipative regime (e.g. the third curve in the left figure) the energy spectrum has about one decade of the $k^{-1 / 2}$ range. This is consistent with the theory. Indeed, according to (14) the non-universal fronts at low and high $k$ ends should be of similar size (on the log scale) as the universal $k^{1 / 2}$ range. In our case, we have about 3.5 decades in total which leaves about one decade for the scaling range.

At later time (in the diffusive regime) one can see that the spectrum acquires a cut-off. In the right-hand figure, the total energy grows exponentially with two different scaling regimes, as expected. The time at which diffusion becomes important is around 4. In agreement with theory, in the diffusive regime the growth rate of the total energy is approximately equal to the growth of the individual $k$-modes. Remarkably, the ratio of the growth rate in the perfect conductor regime to the growth rate in the diffusive regime is in good agreement with theoretical predictions. In Fig. 21 one can see the corresponding mean polarization (left figure) and spectral flatness (right-figure) from this simulation. One can see that the polarization spectrum is flat and it rapidly decreases in time. Both of these features are in agreement with the theoretical KKM results. Thus, the KKM based analytical prediction that the magnetic turbulence becomes plane polarized is much better represented in this simulation than in the previously discussed stochastic simulations. The spectral flatness rapidly grows in time which is also in agreement with the KKM theory. However, there is no obvious region of the theoretical $k^{3 / 2}$ scaling.

\subsection{Code 4}

Code 4 (C4) is almost identical in implementation to $\mathrm{C} 3$. However, in this numerical experiment the strain matrix components were obtained from a $512^{3}$ spectral DNS of the NavierStokes equations at a Taylor Reynolds number of approximately 200 (or 1150 based on the integral scale). The velocity field was forced by keeping the amplitudes of 13 wavenumbers 

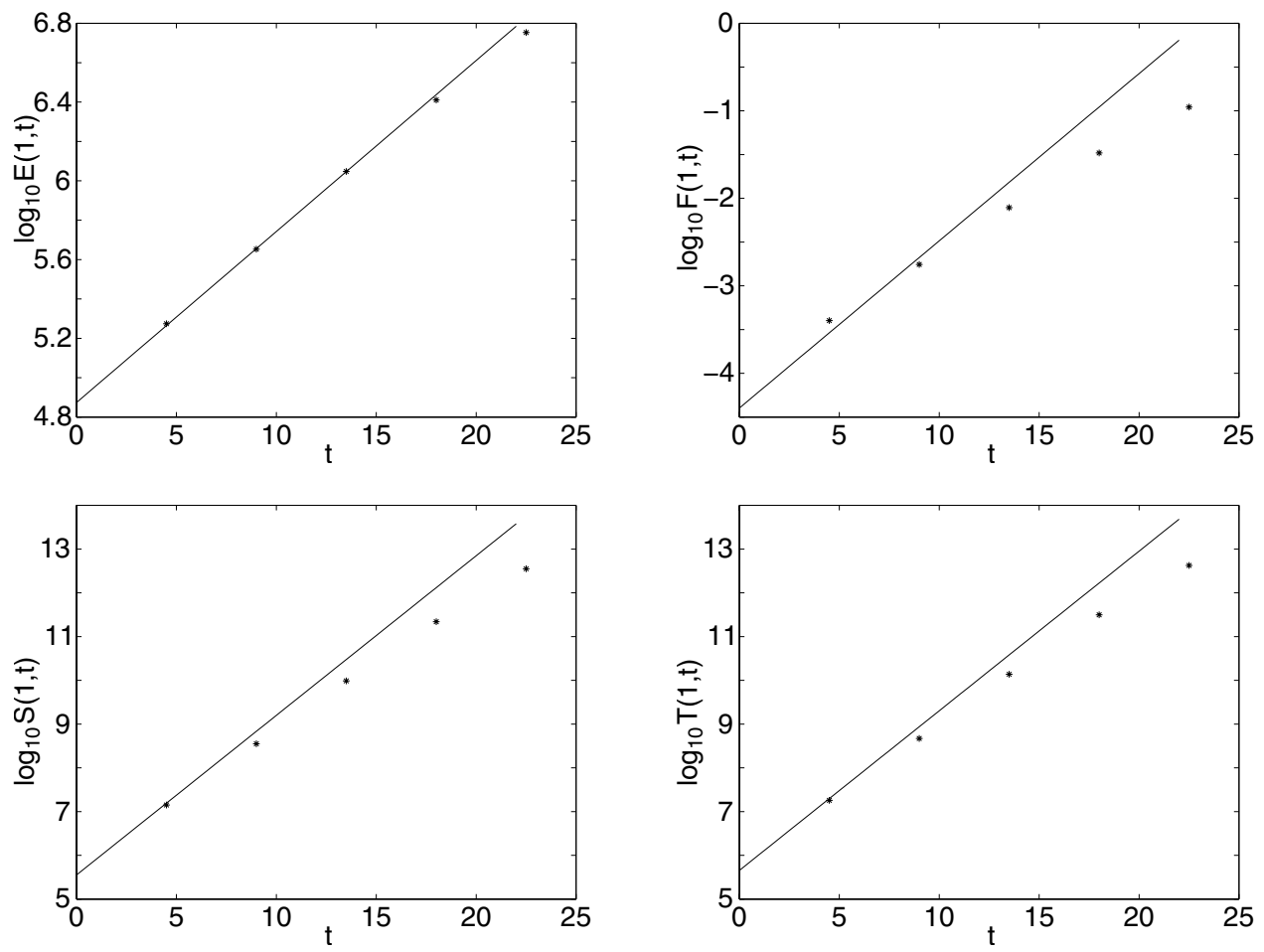

Fig. 15. Growth of $E(\boldsymbol{k}, t)$ (top left), $F(\boldsymbol{k}, t)$ (top right), $S(\boldsymbol{k}, t)$ (bottom left) and $T(\boldsymbol{k}, t)$ (bottom right) in time at $\boldsymbol{k}=1$ for a $\mathrm{C} 2$ simulation with $\Omega=0.36$. The theoretical growth for $E, F, S$ and $T,(14),(27), 22$ and (23) respectively, have also been plotted (solid lines).
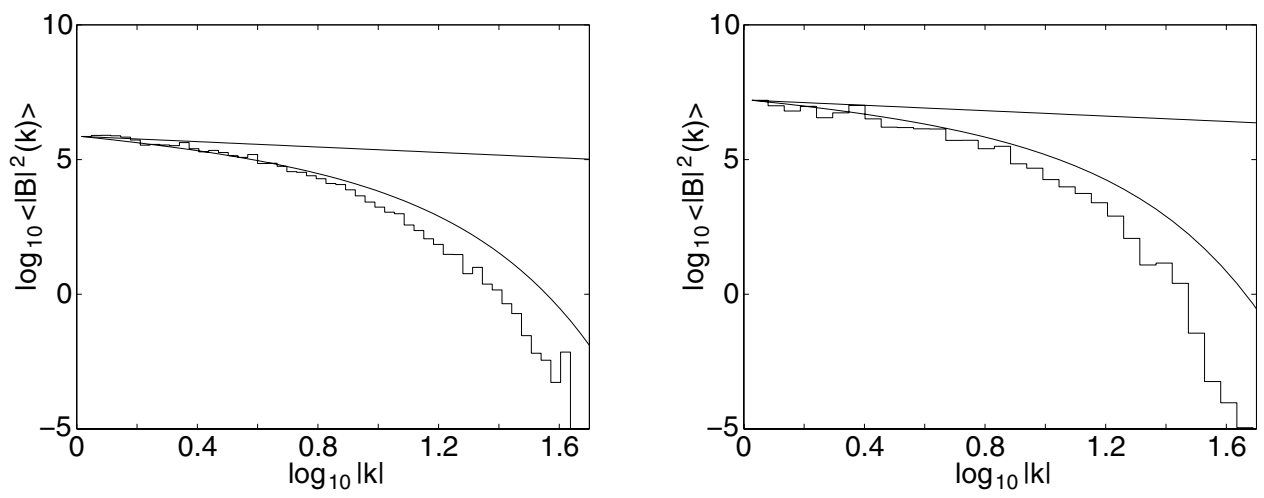

Fig. 16. $E=\left\langle\boldsymbol{B}^{2}(k, t)>\right.$ for a $C 2$ simulation with $\Omega=0.36$ and $\kappa=0.005$. Averaging has been performed over 480000 realizations. The time here is $t=6$ (left figure) and $t=12$ (right figure). The particles have been sorted into 100 bins. The theoretical solution (19) (curve) and a slope of $k^{-1 / 2}$ (straight line) have also been plotted.
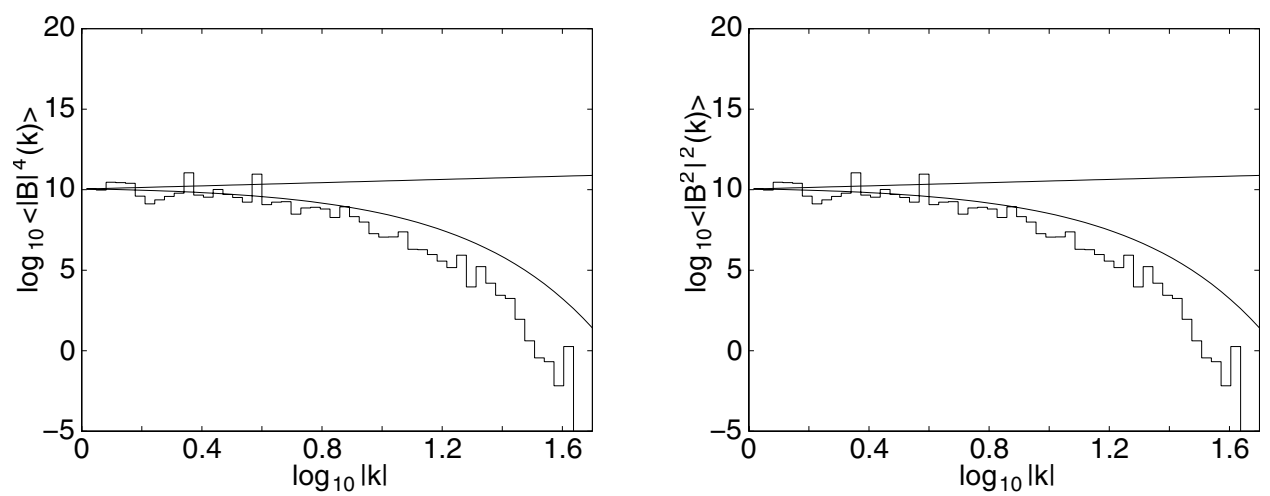

Fig. 17. $S=\left\langle|\boldsymbol{B}|^{4}(k, t)>\right.$ (left figure) and $T=<\left|\boldsymbol{B}^{2}\right|^{2}(k, t)>$ (right figure) for a C2 simulation with $\Omega=0.36$ and $\kappa=0.005$. The time here is $t=6$. Averaging has been performed over 480000 realizations. The particles have been sorted into 100 bins, (but the axis have been re-scaled). The theoretical curves (28) (left figure) and (29) (right figure) as well as a slope of $k^{+1 / 2}$ (straight line) have also been plotted. 


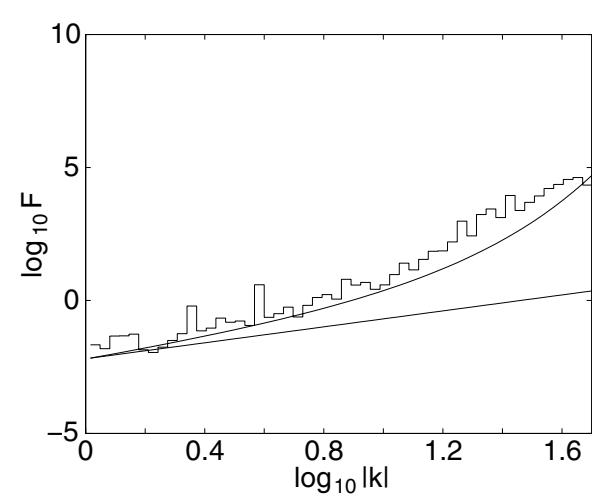

Fig. 18. $F=S / E^{2}$ for a $\mathrm{C} 2$ simulation with $\Omega=0.36, \kappa=0.005$ and $t=6$. Averaging has been performed over 480000 realizations. The particles have been sorted into 100 bins, (but the axis have been rescaled). The theoretical curve (30) and slope $k^{+3 / 2}$ (straight line) have also been plotted.

with modulus $k$ from 0.5 to 1.5 fixed in time. The smallest resolved scale is 0.77 of the Kolmogorov scale (i.e., the DNS is well resolved). The strain time series calculated via recording

$\sigma_{i j}=\frac{\partial v_{i}}{\partial x_{j}}$

along 512 fluid paths. At each of these fluid particles we put 4096 magnetic wavepackets. The rms of the different strain components in this experiment ranged from 6.6 to 9.3 and the correlation time was approximately 0.08 . Thus, the correlation time in this case is of the same order as the inverse strain rate which is natural for real Navier-Stokes turbulence where both values are of the order of the eddy turnover time at the Kolmogorov scale. The timestep in this simulation was taken to be two hundred times smaller than the strain correlation time.

In Fig. 22 we consider the energy spectrum (left figure) and growth of the total energy (right figure). There is a good agreement with the theoretical behavior of the energy spectrum. One can see a region of $k^{1 / 2}$ scaling and a log correction in the diffusive regime. Note that the pure $k^{1 / 2}$ scaling changes to the log-corrected spectrum at the time when the high- $k$ tail hits the dissipative scale which approximately corresponds to the third curve in Fig. 22 measured at $t=0.44$. The growth of the total energy is again well represented. The growth rate exponent changes at around $t=0.4$ which marks the transition between the perfect conductor and the diffusive regimes. In agreement with the theory, the growth of the total energy the diffusive regime is approximately equal to the growth of the energy found in individual $k$ modes. Again the ratio of the growth rates in the perfect conductor and the diffusive regimes is consistent with theoretical predictions.

The $\mathrm{C} 4$ results for the mean polarization and the flatness spectra are shown in Fig. 23. One can see that the mean polarization (left figure) decays in time and is spectrally flat which is in agreement with the KKM theory. The spectral flatness (right figure) grows in time, but the shape of the spectrum is different from the theoretical result of $k^{3 / 2}$. This is similar to the corresponding flatness results obtained in $\mathrm{C} 3$ simulations. In fact, the deviations of the flatness from theory, in both the C3 and C4 simulations, might not be due to changes in strain statistics. The correlation time of the strain in the C3 simulations is short, and therefore one would expect that its numerical results would be closer in behavior to KKM than $\mathrm{C} 4$. However, the degree to which the flatness differs from the KKM results is similar in both the C3 and C4 simulations. Thus, we may conclude that our algorithm is failing to reproduce some features of the higher order correlators.

\section{Discussion}

In this numerical investigation we have used a range of numerical models, based on Lagrangian particle dynamics, to investigate the small-scale turbulent dynamo problem. For the KKM based stochastic models, using a delta-correlated Gaussian strain, we firstly considered the scaling of the even order moments $<\boldsymbol{B}^{2 n}(t)>$ and found good agreement with the theoretical predictions (17) and (18) for the perfect conductor and diffusive regimes respectively. Next we considered various spectral objects including the magnetic energy, polarization and flatness, and made comparisons to their large time asymptotic analytical solutions. Because of the rich analytical results available, the KKM is a good testing ground for our stochastic numerical models. The behavior of the energy spectrum in both diffusive and non-diffusive simulations was found to grow in time and fit the large-time asymptotic forms predicted by theory, with evidence of a $k^{-1 / 2}$ scaling region in each case. The fourth order correlators were also considered. Although noisier than the energy spectrum, these spectra also appeared consistent with the analytical results. However, there was evidence of some hyper-diffusion in these numerical results are high wavenumbers. The new analytical objects, the mean polarization and spectral flatness, were investigated. The flatness was well behaved with an observed $k^{3 / 2}$ scaling and the growth rate in time in agreement with the analytical predictions. In contrast, the polarization of the magnetic turbulence in these simulations was mis-represented. Although it was spectrally flat, in agreement with the theory, it appeared to saturate at its Gaussian value of $1 / 3$ rather than decaying in time. However, on considering individual realizations it was apparent that at least some of the predicted decaying behavior was observed qualitatively in these simulations. This also provided us with a useful visualization of the dynamo system in terms of a cactus leaf in $k$-space. The linear polarization corresponds to the $k$-space alignment of the magnetic field "thorns" along the direction of the smallest Lyapunov exponent, i.e. transverse to the cactus leaf pad. The flatness growth with $k$ is directly linked to the fact that at larger $k$ the cactus leaves will cover the $k$-space more sparsely. We also computed the Lyapunov exponents and found that the largest exponent was well represented which ensures the correct growth of the total magnetic energy. The behavior of the smallest exponent was badly reproduced, which may be connected to the observed behavior of the polarization.

A second set of experiments was also performed to test the universality of the KKM based theoretical results with respect to changes in the strain statistics. These two simulations, used a finite-correlated Gaussian and a DNS generated strain fields respectively and their results showed an agreement with theory for both the growth rate of the total energy and the $k^{-1 / 2}$ 

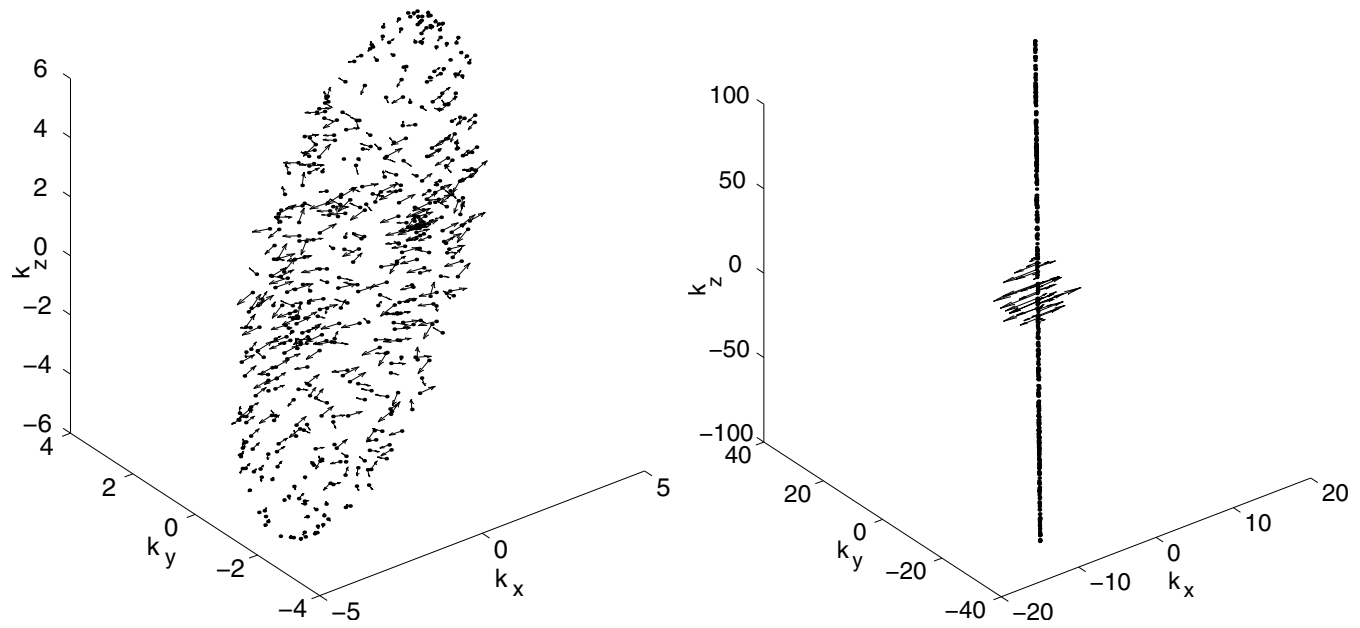

Fig. 19. The magnetic field of 500 wavepackets in a $C 2$ simulation with $\Omega=0.36$ and $\kappa=0.005$. The figures show the particle's positions in $k$-space at $t=6$ and their corresponding real magnetic fields for two different realizations of the strain matrix. The left figure corresponds to the same strain field as the left-hand graph of Fig. 12 and similarly for the right-hand graphs.
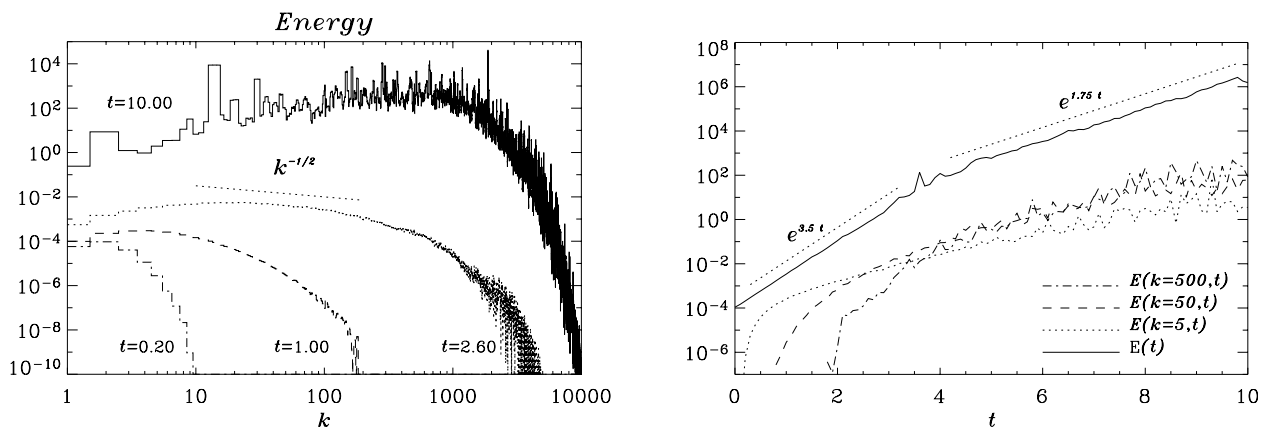

Fig. 20. The magnetic energy spectrum $E(k, t)$ (left figure) and growth of the total magnetic energy $<\boldsymbol{B}^{2}(t)>$ (right figure) for the case of a synthetic Gaussian strain field with finite correlation time. The right-hand figure also shows the growth of the energy for a selection of individual $k$-modes.
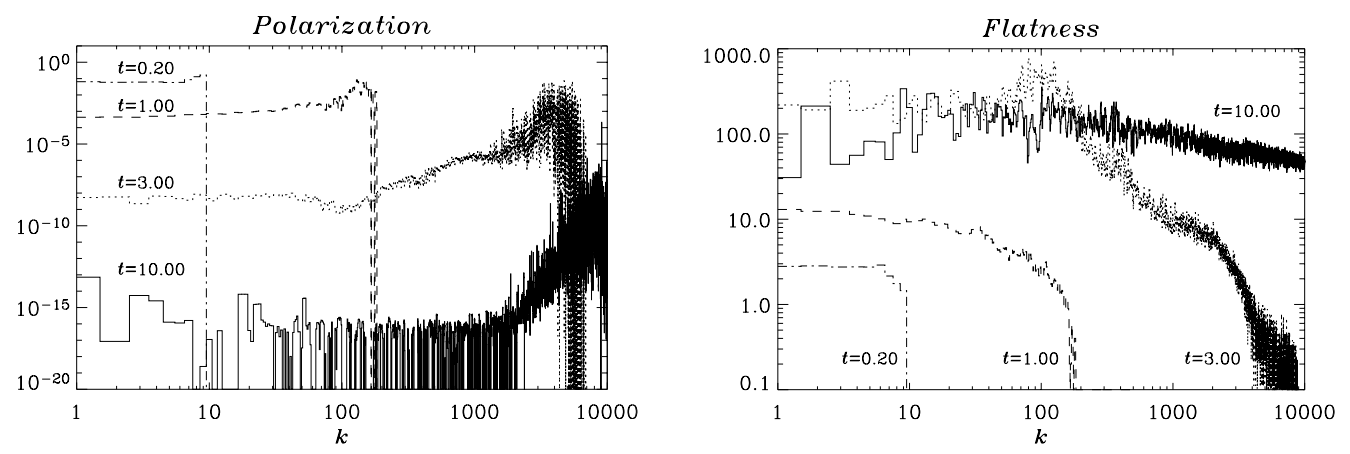

Fig. 21. The normalized mean polarization (left figure) and spectral flatness (right figure) for the case of a synthetic Gaussian strain field with finite correlation time.

scaling. Note that a clear $k^{-1 / 2}$ scaling was also recently observed in full DNS of the MHD equations even for order one Prandtl numbers Haugen et al. (2003). Further, both of our numerical experiments demonstrated excellent agreement with the theoretical behavior of the polarization, in each case being both spectrally flat and decaying in time. Also in agreement with the theory is the rapid time growth of the flatness. In contrast, the spectral shape of the flatness in these simulations was not as well represented as in the stochastic based results. For both the finite-correlated Gaussian and DNS strain based simulations the flatness spectrum was far from the predicted $k^{3 / 2}$ scaling. We believe this difference from theory is a numerical artifact from our algorithm, rather than directly due to changes in the form of the velocity field. We conclude that the gross features predicted by the KKM theory seem to be robust and insensitive to changes in the strain statistics.

Interestingly, Brandenburg et al. (1995), and more recently Schekochihin et al. (2001); Schekochihin et al. (2002b), investigated the behavior of the real space curvature of the field $\boldsymbol{C}=\boldsymbol{b} \cdot \nabla \boldsymbol{b}$ where $\boldsymbol{b}=\boldsymbol{B} /|\boldsymbol{B}|$. They found that the 

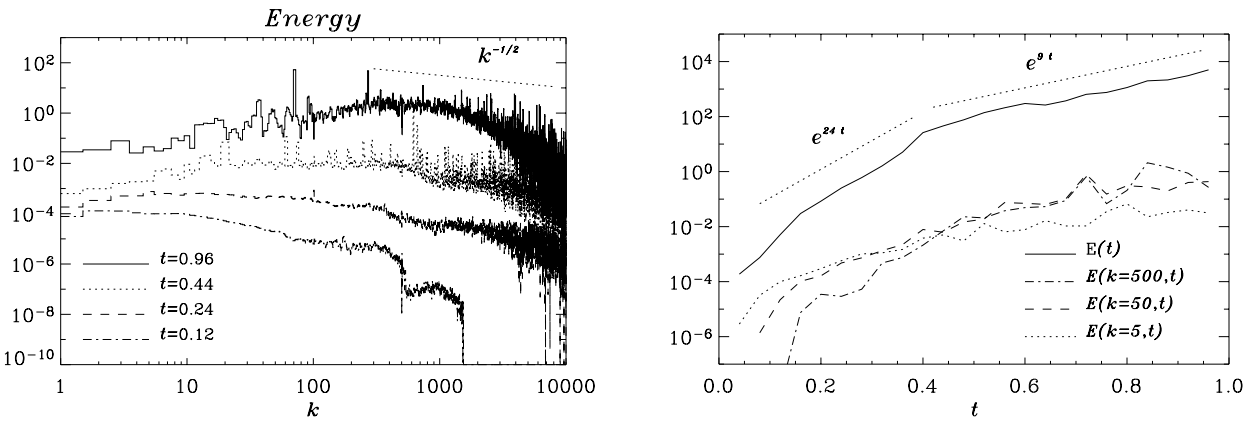

Fig. 22. The magnetic energy spectrum $E(k, t)$ (left figure) and growth of the total magnetic energy $<\boldsymbol{B}^{2}(t)>$ (right figure) for the case of a strain obtained from a $512^{3}$ DNS. The right-hand figure also shows the growth of the energy for a selection of individual $k$-modes.
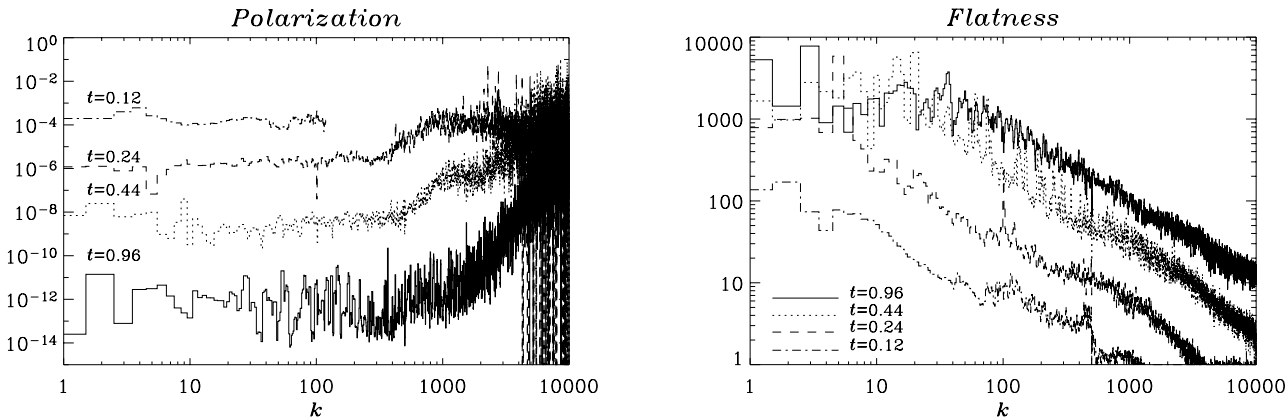

Fig. 23. The normalized mean polarization (left figure) and spectral flatness (right figure) for the case of a strain obtained from a $512^{3}$ DNS.

curvature of the magnetic field is anti-correlated with its strength, which corresponds to folded and strongly stretched structures. This agrees with our result that the Fourier modes of the magnetic field tend to a state of plane polarization. However, it should be noted that the Fourier polarization gives more information than the curvature statistics. Indeed, the zero curvature requirement allows both layered sheets and filamentary tubes. Further, layered magnetic field may vary its direction as one passes from one sheet to another. Our analysis narrows down the choice and indicates that the magnetic field structures are sheets in coordinate space, thus ruling out the existence of any thin tube structures. Further, the plane polarization result also eliminates the possibility of any "twists" in the magnetic field between the individual sheets. That is, the sheet magnetic field is aligned along the same direction. Although the field in successive sheets can be parallel or anti-parallel to each other. In fact, the presence of one neutral direction in the Lagrangian deformations tells us that the layers have a finite width in one direction and thus look, in coordinate space, like ribbons with the magnetic field lying along these ribbons.

Acknowledgements. We would like to thank Warwick University's Fluid Dynamic Research Centre for the use of their computer facilities. The simulations with the code $\mathrm{C} 4$ were performed at IDRIS (France).

\section{Appendix}

In general, the strain matrix can be expressed as

$<\sigma_{i j}(t) \sigma_{k l}(0)>=C_{1}\left(\delta_{i k} \delta_{j l}+C_{2} \delta_{i j} \delta_{k l}-\left(1+C_{2}\right) \delta_{i l} \delta_{j k}\right) \delta(t),(39)$ where $C_{i}$ are constants (see for example McComb 1990). Written in this way the strain statistics automatically satisfy the incompressibility condition.

For our particular choice (12), we have,

$<\sigma_{i j}(t) \sigma_{k l}(0)>=\Omega\left(\delta_{i k} \delta_{j l}-\frac{1}{3} \delta_{i j} \delta_{k l}\right) \delta(t)$,

giving $C_{1}=\Omega$ and $C_{2}=-1 / 3$. In contrast, the covariance chosen by Chertkov et al. (1999) and Falkovich et al. (2001) is

$<\sigma_{i j}(t) \sigma_{k l}(0)>=\frac{\lambda_{1}}{3}\left(4 \delta_{i k} \delta_{j l}-\delta_{i j} \delta_{k l}-\delta_{i l} \delta_{j k}\right) \delta(t)$,

corresponding to $C_{1}=4 \lambda_{1} / 3$ and $C_{2}=-1 / 4$, where $\lambda_{1}$ is the growth rate of a material line element in the flow. Setting $i=k=\alpha$ and $j=l=\beta$ in both (40) and (41) we find,

$<\sigma_{\alpha \beta}(t) \sigma_{\alpha \beta}(0)>=8 \Omega \delta(t)$

$<\sigma_{\alpha \beta}(t) \sigma_{\alpha \beta}(0)>=10 \lambda_{1} \delta(t)$

The latter expression is the previous stated Chertkov et al. (1999) formalism (9) from Sect. 2.

\section{References}

Balkovsky, E., \& Fouxon, A. 1999, Phys. Rev. E, 60(4), 4164

Batchelor, G. K. 1950, Proc. Roy. Soc. A, 202, 405

Batchelor, G. K. 1954, Proc. Roy. Soc., 2

Brandenburg, A., Procaccia, I., \& Segel, D. 1995, Phys. Plasmas, 2, 1148

Chandran, B. D. G. 1998, ApJ, 492, 179

Chertkov, M., Falkovich, G., Kolokolov, I., \& Vergassola, M. 1999, Phys. Rev. Lett., 83(20), 4065 
Childress, S., \& Gilbert, A. 1995 Stretch, Twist, Fold: The Fast Dynamo (Berlin: Springer-Verlag)

Falkovich, G., Gawedzki, K., \& Vergassola, M. 2001, Rev. Mod. Phys., 73, 913

Goldhirsch, I., Sulem, P.-L., \& Orszag, S. A. 1987, Physica D, 27, 311

Haugen, N., Brandenburg, A., \& Dobler, W. 2003 [astro-ph/0307059]

Kazantsev, A. P. 1968, Sov. Phys. JETP, 26, 1031

Kloeden, P. E., Platen, E., \& Schurz, H. 1997, Numerical Solution of SDE Through Computer Experiments (Berlin: Springer-Verlag)

Kraichnan, R. H., \& Nagarajan, S. 1967, Phys. Fluids, 10, 859

Kraichnan, R. H. 1974, J. Fluid Mech., 64, 737

Kulsrud, R., \& Anderson, S. 1992, ApJ, 396, 606

Kulsrud, R. 1999, ARA\&A, 37, 37

McComb, W. D. 1990, The Physics of Fluid Turbulence (Oxford: Clarendon Press)

Moffatt, H. K. 1978, Magnetic Field Generation in Electrically Conducting Fluids (Cambridge: Cambridge University Press)

Nazarenko, S. V., West, R. J., \& Zaboronski, O. 2003, Phys. Rev. E, 68, 026311 (also [astro-ph/0304540] v1 29 Apr. 2003)
Novikov, V. G., Ruzmaikin, A. A., \& Sokoloff, D. D. 1983, JETP, 58, 527

Parker, E. N. 1979, Cosmic Magnetic Field, Their Origin and Activity (Oxford: Clarendon Press)

Press, W. H., Falnnery, B. P., Teukolsky, S. A., \& Vetterling, W. T. 1993, Numerical Recipes in C (Cambridge: Cambridge University Press)

Soward, A. M. 1994, Fast Dynamos, Lectures on Solar and Planetary Dynamos, Chap. 6, ed. M. R. E. Proctor, \& A. D. Gilbert (Cambridge: Cambridge University Press)

Schekochihin, A. A., Cowley, S., \& Maron, J. L. 2001, Phys. Rev. E., 65,016305

Schekochihin, A. A., Boldyrev, S. A., \& Kulsrud, R. M. 2002a, ApJ, 567,828

Schekochihin, A. A., Maron, J. L., Cowley, S., \& McWilliams, J. C. 2002b, ApJ, 576, 806

Zel'dovich, Y. B., Ruzmaikan, A. A., Molchanov, S. A., \& Sokoloff, D. D. 1984, J. Fluid Mech., 144, 1 